\title{
THE X-RAY PROPERTIES OF MILLION SOLAR MASS BLACK HOLES
}

\author{
Richard. M. Plotkin ${ }^{1,2}$, Elena Gallo ${ }^{1}$, Francesco Haardi ${ }^{3,4}$, Brendan P. Miller ${ }^{5}$, Callum J. L. Wood ${ }^{2}$, \\ Amy E. Reines ${ }^{1,6,8}$, Jianfeng Wu ${ }^{1}$, and Jenny E. GReEne ${ }^{7}$ \\ ${ }^{1}$ Department of Astronomy, University of Michigan, 1085 South University Avenue, Ann Arbor, MI 48109, USA; richard.plotkin@curtin.edu.au \\ ${ }^{2}$ International Centre for Radio Astronomy Research (ICRAR), Curtin University, G.P.O. Box U1987, Perth, WA 6845, Australia \\ ${ }^{3}$ DiSAT, Università dell'Insubria, via Valleggio 11 , I-22100 Como, Italy \\ ${ }^{4}$ INFN, Sezione di Milano-Bicocca, Piazza della Scienza 3, I-20126 Milano, Italy \\ ${ }^{5}$ Department of Chemistry and Physical Sciences, The College of St. Scholastica, Duluth, MN 55811, USA \\ ${ }^{6}$ National Optical Astronomy Observatory, 950 North Cherry Avenue, Tucson, AZ 85719, USA \\ ${ }^{7}$ Department of Astrophysics, Princeton University, Princeton, NJ 08544, USA \\ Received 2015 October 27; revised 2016 April 21; accepted 2016 April 29; published 2016 July 12
}

\begin{abstract}
We present new Chandra X-ray observations of seven low-mass black holes $\left(M_{\mathrm{BH}} \approx 10^{6} M_{\odot}\right)$ accreting at lowbolometric Eddington ratios between $-2.0 \lesssim \log L_{\mathrm{bol}} / L_{\mathrm{Edd}} \lesssim-1.5$. We compare the X-ray properties of these seven low-mass active galactic nuclei (AGNs) to a total of 73 other low-mass AGNs in the literature with published Chandra observations (with Eddington ratios extending from $-2.0 \lesssim \log L_{\mathrm{bol}} / L_{\mathrm{Edd}} \lesssim-0.1$ ). We do not find any statistical differences between the low and high Eddington ratio low-mass AGNs in the distributions of their X-ray to ultraviolet luminosity ratios $\left(\alpha_{\mathrm{ox}}\right)$, or in their X-ray spectral shapes. Furthermore, the $\alpha_{\mathrm{ox}}$ distribution of low$L_{\text {bol }} / L_{\text {Edd }}$ AGNs displays an X-ray weak tail that is also observed within high- $L_{\text {bol }} / L_{\text {Edd }}$ objects. Our results indicate that between $-2 \lesssim \log L_{\mathrm{bol}} / L_{\mathrm{Edd}} \lesssim-0.1$, there is no systematic change in the structure of the accretion flow for active galaxies hosting $10^{6} M_{\odot}$ black holes. We examine the accuracy of current bolometric luminosity estimates for our low- $L_{\mathrm{bol}} / L_{\mathrm{Edd}}$ objects with new Chandra observations, and it is plausible that their Eddington ratios could be underestimated by up to an order of magnitude. If so, then in analogy with weak emission line quasars, we suggest that accretion from a geometrically thick, radiatively inefficient "slim disk" could explain their diverse properties in $\alpha_{\mathrm{ox}}$. Alternatively, if current Eddington ratios are correct (or overestimated), then the X-ray weak tail would imply that there is diversity in disk/corona couplings among individual low-mass objects. Finally, we conclude by noting that the $\alpha_{\mathrm{ox}}$ distribution for low-mass black holes may have favorable consequences for the epoch of cosmic reionization being driven by AGN.
\end{abstract}

Key words: accretion, accretion disks - galaxies: active - X-rays: galaxies

\section{INTRODUCTION}

Virtually every large galaxy harbors a supermassive black hole (SMBH; $M_{\mathrm{BH}} \approx 10^{6}-10^{9} M_{\odot}$ ) in its nucleus (e.g., Kormendy \& Ho 2013). However, understanding in detail the mechanism(s) that formed the first primordial "seed" black holes, and determining the evolutionary path that those seeds took to grow to SMBH sizes, remains a fundamental problem (e.g., Greene 2012; Natarajan 2014). We currently know of $\sim 40$ quasars at $z \gtrsim 6$ with $>10^{9} M_{\odot}$ SMBHs (see, e.g., Willott et al. 2003; Fan et al. 2006; Wu et al. 2015). Black hole seeds produced by the collapse of Population III stars would have $M_{\mathrm{BH}} \lesssim 1000 M_{\odot} \quad$ (e.g., Madau \& Rees 2001; Bromm et al. 2002), and one generally needs to invoke superEddington accretion onto such low-mass seed black holes to explain the presence of high-redshift quasars (Madau et al. 2014). On the other hand, more massive seeds $\left(10^{5}-10^{6} M_{\odot}\right)$, as could be produced from the direct collapse of gas clouds at the centers of galaxies (e.g., Begelman et al. 2006; Lodato \& Natarajan 2006), provide another feasible way to cultivate rapid growth. We refer to $10^{5}-10^{6} M_{\odot}$ black holes as "massive black holes" (mBHs).

Over the past 10-15 years, samples of $\mathrm{mBH}$-powered active galactic nuclei (AGNs) have rapidly evolved from discoveries of a handful of individual objects-such as NGC 4395 (Filippenko \& Ho 2003), POX 52 (Barth et al. 2004), and Henize 2-10 (Reines et al. 2011) - to the production of

\footnotetext{
${ }^{8}$ Hubble Fellow.
}

systematically assembled catalogs drawn from large sky surveys (e.g., Greene \& Ho 2007a; Barth et al. 2008; Dong et al. 2012b; Reines et al. 2013; Schramm et al. 2013; Moran et al. 2014). The largest $\mathrm{mBH}$ catalogs so far have utilized the Sloan Digital Sky Survey (SDSS; York et al. 2000), particularly Greene \& Ho (2007, hereafter GH07) and Dong et al. (2012b) who select mBH AGNs based on the presence of broad $\mathrm{H} \alpha$ emission in optical spectra. They use the widths and luminosities of broad $\mathrm{H} \alpha$ to estimate black hole masses and bolometric luminosities via virial scaling techniques (Greene \& Ho 2005). Both studies require $M_{\mathrm{BH}}<2 \times 10^{6} M_{\odot}$, and their catalogs include 229 (GH07) and 309 (Dong et al. 2012b) objects. Reines et al. (2013) also recover a sizable number of $\mathrm{mBH}$ candidates $(151$ objects) with a slightly different approach. They specifically target dwarf galaxies by restricting host galaxy stellar masses to $M_{\star}<3 \times 10^{9} M_{\odot}$. The vast majority (136) of their $\mathrm{mBH}$ AGN candidates are then selected via photoionization signatures of black hole activity via narrow emission line ratios (only a small fraction of their 151 AGN candidates display broad $\mathrm{H} \alpha$ ). The smallest $\mathrm{mBH}$ discovered so far through optical searches weighs in at only $5 \times 10^{4} M_{\odot}$ (Reines et al. 2013; Baldassare et al. 2015).

It is natural to turn to the $\mathrm{X}$-ray waveband for insights into the properties of the accretion flows that feed $\mathrm{mBHs}$, because $\mathrm{X}$-ray emission is a nearly universal feature of accretion. So far, the most extensive X-ray follow-up is based on 67 objects that were observed with the Chandra X-ray telescope (Greene \& Ho 2007c; Desroches et al. 2009; Dong et al. 2012a, 
Table 1

Galaxy Properties and X-Ray Observing Log

\begin{tabular}{|c|c|c|c|c|c|c|c|}
\hline $\begin{array}{l}\text { Galaxy Name } \\
\text { (SDSS J) } \\
\text { (1) }\end{array}$ & $\begin{array}{c}\text { GH07 ID } \\
\text { (2) }\end{array}$ & $\begin{array}{l}z \\
(3)\end{array}$ & $\begin{array}{c}\log N_{\mathrm{H}, \mathrm{gal}} \\
\left(\mathrm{cm}^{-2}\right) \\
(4)\end{array}$ & $\begin{array}{c}\log l_{2500} \\
\operatorname{erg~s}^{-1} \mathrm{~Hz}^{-1} \\
\text { (5) }\end{array}$ & $\begin{array}{c}\log M_{\mathrm{BH}} \\
\left(M_{\odot}\right) \\
(6)\end{array}$ & $\begin{array}{c}\log \left(L_{\mathrm{bol}} / L_{\mathrm{Edd}}\right) \\
\text { (7) }\end{array}$ & $\begin{array}{l}\text { ObsID } \\
\text { (8) }\end{array}$ \\
\hline $003552.26+011249.4$ & 7 & 0.0414 & 20.4 & 26.3 & 5.8 & -1.7 & 15015 \\
\hline $081825.15+472950.3$ & 40 & 0.0537 & 20.6 & 26.7 & 6.0 & -1.5 & 15016 \\
\hline $083803.67+540642.2$ & 53 & 0.0295 & 20.6 & 26.6 & 6.2 & -1.8 & 15018 \\
\hline $084013.23+412357.0$ & 56 & 0.0290 & 20.3 & 26.4 & 6.2 & -2.0 & 15019 \\
\hline
\end{tabular}

Note. Column (1) SDSS galaxy designation. Column (2) galaxy ID from GH07. Column (3) spectroscopic redshift from the SDSS. Column (4) log galactic column density along line of sight from Dickey \& Lockman (1990) H I maps. Column (5) log AGN continuum luminosity density at 2500 A (see Section 3.2). Column (6) log of the virial-scaled $\mathrm{H} \alpha$-based black hole mass from GH07. Column (7) log Eddington ratio from GH07. Column (8) Chandra Observation ID. Column (9) effective exposure time of Chandra observation.

hereafter D12; Ludlam et al. 2015; also see Dewangan et al. 2008; Miniutti et al. 2009, for an XMM-Newton view of about a dozen mBH-powered AGN). These X-ray observations show that GH07 mBHs tend to have higher X-ray to ultraviolet (UV) luminosity ratios, on average, compared with $\left(10^{8}-10^{9} M_{\odot}\right)$ Type-1 quasars. The higher X-ray to UV ratios are generally consistent with expectations from accretion disk/ corona models (e.g., Haardt \& Maraschi 1993; Done et al. 2012) where lower black hole masses yield higher accretion disk temperatures, which results in less UV emission near $2500 \AA$ (combined with less efficiently Compton-cooled coronae, this yields higher X-ray to UV flux ratios; see D12). However, as a population, mBH AGNs show a large dispersion in their X-ray to UV luminosity ratios, and they display a puzzling "X-ray weak" tail (D12).

The aforementioned X-ray studies only targeted $\mathrm{mBHs}$ at high Eddington ratios ${ }^{9}\left(\log L_{\mathrm{bol}} / L_{\mathrm{Edd}} \gtrsim-1\right)$. Here, we present new X-ray observations of seven GH07 mBHs at Eddington ratios up to an order of magnitude lower $\left(-2.0 \lesssim \log L_{\text {bol }} / L_{\text {Edd }} \lesssim-1.5\right)$. By increasing the dynamic range in Eddington ratio, we can search for trends between X-ray properties and $L_{\text {bol }} / L_{\text {Edd }}$. We describe our sample selection and X-ray observations/analysis in Section 2. X-ray results are presented in Section 3, where we also discuss the optical emission line properties of our targets (Section 3.3), as well as bolometric corrections for mBHs (Section 3.4). Our results are discussed in Section 4. For consistency with previous Chandra follow-ups, all optical spectroscopic measurements are taken from GH07, and we generally follow the recipes of D12 when deriving X-ray properties. We adopt the same cosmology as GH07 and D12: $H_{0}=71 \mathrm{~km} \mathrm{~s}^{-1} \mathrm{Mpc}^{-1}$, $\Omega_{m}=0.27$, and $\Omega_{\Lambda}=0.75$ (Spergel et al. 2003).

\section{SAMPLE AND OBSERVATIONS}

Chandra targets were selected from the GH07 catalog of mBHs, from which we consider low Eddington ratio AGNs with $\log L_{\mathrm{bol}} / L_{\mathrm{Edd}}<-1.5$ (for estimating bolometric luminosities, GH07 assume that $L_{\mathrm{bol}}=9.8 L_{5100}$, where $L_{5100}$

\footnotetext{
$9 \quad L_{\text {bol }}$ is the bolometric luminosity, which GH07 calculate based on the luminosity of broad $\mathrm{H} \alpha$ emission (see their Section 3). The Eddington luminosity, $L_{\mathrm{Edd}}=1.26 \times 10^{38}\left(M / M_{\odot}\right) \mathrm{erg} \mathrm{s}^{-1}$ for ionized Hydrogen, is the maximum luminosity before radiation pressure halts accretion, assuming a spherical geometry and isotropic radiation.
}

is the AGN luminosity at $5100 \AA$; please refer to Sections 3.2 and 3.4 for details). There are $17 \mathrm{mBHs}$ in $\mathrm{GH} 07$ with such low Eddington ratios. To improve X-ray efficiency, we further restrict our target list to only include nearby galaxies $(z<0.055$, which is comparable to the average redshift of GH07 objects targeted by D12), providing seven Chandra targets. These seven galaxies were observed by Chandra during cycle-14 (proposal ID 14700673; PI Gallo). The galaxy properties and observations are summarized in Table 1. Throughout the text, we refer to each galaxy by the catalog number assigned by GH07 (see Column 2 of Table 1).

\subsection{X-Ray Analysis}

Each galaxy was placed at the aimpoint of the S3 chip on the Advanced CCD Imaging Spectrometer (ACIS; Garmire et al. 2003). The data were telemetered in FAINT mode and reduced with the Chandra Interactive Analysis of Observations software v4.7 (CIAO; Fruscione et al. 2006). We reprocessed each Chandra observation, applying the latest calibration files (CALDB v4.6.7). The event files were then filtered to only retain events with grades $0,2,3,4$, and 6 , and energies between $0.3-8 \mathrm{keV}$. We examined light curves of the ACIS background and removed time periods where the background deviated by more than $3 \sigma$ from the mean, using the lc_sigma_clip routine in CIAO. Only $200 \mathrm{~s}$ were removed from ObsIDs 15015 and 15016 (galaxies 7 and 40), and $400 \mathrm{~s}$ were removed from ObsID 15020 (galaxy 82). The effective exposure times for each observation are listed in Table 1.

We ran the automated point-source detection tool wavdetect on $0.3-8 \mathrm{keV}$ images of the $\mathrm{S} 3$ chip, adopting wavelet scales of $1.0,1.4,2.0,2.8$, and 4.0 pixels, a point-spread function map calculated at $2.3 \mathrm{keV}$, and a significance threshold of $10^{-6}$ (which corresponds to one expected false detection across the S3 chip). Within the positional accuracy of a source located at the aimpoint of the ACIS detector ( $\sim$ ". 4$)$, four observations contained an X-ray point source at a location consistent with the position of the SDSS optical galaxy (galaxies 40, 53, 56, and 111). Images of each Chandra target (over $0.5-8 \mathrm{keV}$ ) are displayed in Figure 1. All X-ray sources are point-like, with no signs of nearby diffuse emission or nearby (off-nuclear) neighbors. We associate all four X-ray detections with the AGNs (see Sections 3). 

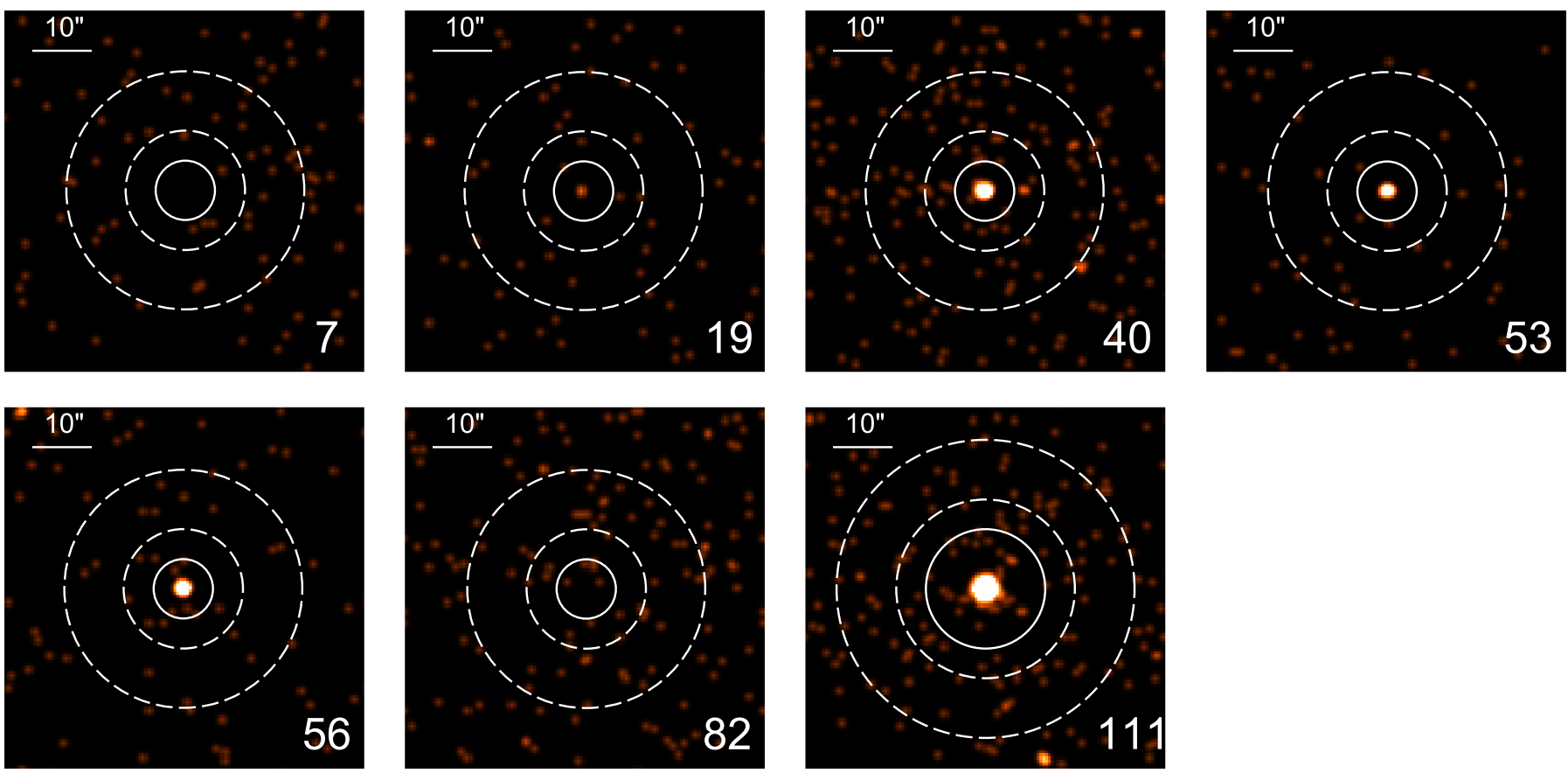

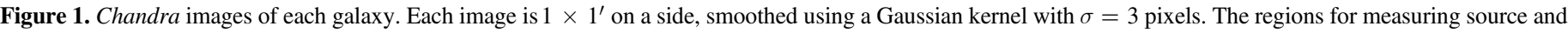

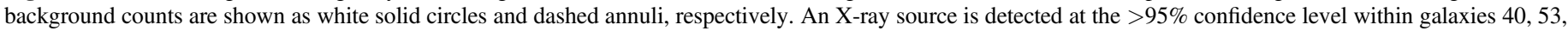
56 , and 111.

Table 2

X-Ray Properties

\begin{tabular}{|c|c|c|c|c|c|c|c|c|}
\hline $\begin{array}{l}\text { GH07 ID } \\
\text { (1) }\end{array}$ & $\begin{array}{c}N_{s} \\
\text { (counts) } \\
(2)\end{array}$ & $\begin{array}{c}N_{h} \\
\text { (counts) } \\
(3)\end{array}$ & $\begin{array}{c}R_{s} \\
(\text { counts ks } \\
(4)\end{array}$ & $\begin{array}{c}R_{h} \\
\left(\text { counts ks }{ }^{-1}\right) \\
(5)\end{array}$ & $\begin{array}{c}\log f_{s, u} \\
\left(\mathrm{erg} \mathrm{s}^{-1} \mathrm{~cm}^{-2}\right) \\
(6)\end{array}$ & $\begin{array}{c}\log f_{h, u} \\
\left(\operatorname{erg~s}^{-1} \mathrm{~cm}^{-2}\right) \\
(7)\end{array}$ & $\begin{array}{c}\log L_{s} \\
\left(\mathrm{erg} \mathrm{s}^{-1}\right) \\
(8)\end{array}$ & $\begin{array}{c}\log L_{h} \\
\left(\operatorname{erg~s}^{-1}\right) \\
(9)\end{array}$ \\
\hline 7 & $\ldots$ & $\ldots$ & $<0.2$ & $<0.3$ & $<-14.9$ & $<-14.3$ & $<39.7$ & $<40.3$ \\
\hline 19 & $\ldots$ & $\ldots$ & $<0.3$ & $<0.4$ & $<-14.8$ & $<-14.2$ & $<39.5$ & $<40.2$ \\
\hline 40 & $54.7 \pm 12.7$ & $25.2 \pm 8.9$ & $2.2 \pm 0.5$ & $1.0 \pm 0.4$ & $-14.0 \pm 0.1$ & $-13.7 \pm 0.2$ & 40.8 & 41.1 \\
\hline 53 & $12.8 \pm 5.3$ & $14.2 \pm 5.6$ & $1.4 \pm 0.6$ & $1.6 \pm 0.6$ & $-13.4 \pm 0.2$ & $-13.5 \pm 0.2$ & 40.9 & 40.8 \\
\hline 56 & $38.5 \pm 10.8$ & $18.3 \pm 7.7$ & $4.3 \pm 1.2$ & $2.1 \pm 0.9$ & $-13.7 \pm 0.1$ & $-13.4 \pm 0.2$ & 40.6 & 40.9 \\
\hline 82 & $\ldots$ & $\ldots$ & $<0.2$ & $<0.2$ & $<-15.0$ & $<-14.4$ & $<39.7$ & $<40.4$ \\
\hline 111 & $315.7 \pm 29.8$ & $791.1 \pm 46.8$ & $14.5 \pm 1.4$ & $36.3 \pm 2.2$ & $-12.3 \pm 0.04$ & $-12.0 \pm 0.03$ & 42.4 & 42.8 \\
\hline
\end{tabular}

Note. Column (1) GH07 galaxy ID. Column (2) net counts in the soft energy band (0.5-2 keV). Error bars are at the 90\% confidence level, assuming Poisson statistics (Gehrels 1986). Blank values denote non-detections. Column (3) net counts in the hard energy band (2-8 keV). Column (4) net count rate in the soft energy band (0.5-2 keV). Upper limits are provided for non-detections (95\% level). Column (5) net count rate in the hard energy band (2-8 keV). Column (6) logarithm of unabsorbed soft X-ray flux (0.5-2 keV), assuming an absorbed power-law model. If available, we adopt the best-fit column densities and photon indices in Table 3. Otherwise, we use the Galactic column density in Table 1 and $\Gamma=2$ (the average photon index for GH07 AGNs in D12). Error bars are based on propagating the statistical errors on the net count rates, and they do not include errors on the emission model parameters. All fluxes and luminosities for galaxy 111 are corrected for pileup. Column (7) logarithm of unabsorbed hard X-ray flux (2-8 keV). Column (8) logarithm of unabsorbed luminosity in the soft band (0.5-2 keV). Column (9) logarithm of unabsorbed luminosity in the hard band $(2-8 \mathrm{keV})$.

Next, we created soft- $(0.5-2 \mathrm{keV})$ and hard-band (2-8 keV) images of the S3 chip (these bands were chosen to match the analysis of D12). The numbers of soft and hard X-ray counts were extracted over circular regions with 5" radii, centered on the nuclear X-ray source (or the SDSS optical position for the three observations where wavdetect did not find an X-ray source). The local background was estimated over an annulus concentric with the source extraction region, with inner and outer radii of $10^{\prime \prime}$ and $20^{\prime \prime}$, respectively. The X-ray source in galaxy 111 is significantly brighter than the others, so we adopted a $10^{\prime \prime}$ radius source extraction circle, and a background annulus with inner and outer radii $15^{\prime \prime}$ and $25^{\prime \prime}$ for that observation. In Table 2, we list the total numbers of counts extracted within each circular region, and the net count rates in both the soft and hard bands. We typically obtained $\approx 10-50$ net counts in each band, except for galaxy 111 where we obtained $\approx 300$ and 800 net soft- and hard counts, respectively.

For the four galaxies with associated X-ray emission, we assign uncertainties on the total numbers of counts using Poisson statistics (Gehrels 1986). For the other three galaxies, we use the estimated number of background counts in each source aperture (scaled from the measured number of background counts in each sky annulus) and the Bayesian method of Kraft et al. (1991) to calculate the number of counts required to detect an X-ray source at the $95 \%$ confidence level (typically 3-6 counts). We confirm that no X-ray point source is present for these three galaxies, and we include $95 \%$ upper limits on the net count rates in Table 2. 
Finally, we estimate unabsorbed X-ray fluxes (and luminosities) using the Portable, Interactive Multi-Mission Simulator (PIMMS) $^{10}$ and a power-law model, where we adopt the bestfit photon index $(\Gamma)$ and column density $\left(N_{\mathrm{H}}\right)$ described in Section 2.2. For the three galaxies lacking an X-ray source, we provide flux and luminosity upper limits by assuming $\Gamma=2.0$ (the average value from D12) and the Galactic $N_{\mathrm{H}}$ from the Dickey \& Lockman (1990) H I maps (see Table 1).

\subsection{X-Ray Spectral Analysis}

For the four galaxies with nuclear X-ray detections, we extract X-ray spectra over $0.3-8 \mathrm{keV}$ using the CIAO tool specextract. We use the Interactive Spectral Interpretation System (ISIS; Houck \& Denicola 2000) to analyze the spectra. Given the typically low number of counts, and to allow for comparison with the spectral analysis of D12, we only attempt to fit an absorbed power-law model to each spectrum (phabs*powerlaw in ISIS). We utilize Cash statistics (Cash 1979), with the background included in each spectral fit.

The spectral fits are shown in Figure 2, and the best-fit spectral parameters are reported in Table 3, including the photon index $(\Gamma)$ and column density $\left(N_{\mathrm{H}}\right)$. Error bars are quoted at the $90 \%$ level (corresponding to a change in the Cash statistic of $\Delta C=2.71$ for one parameter of interest). Two sources (galaxies 53 and 111) display moderate absorption, with intrinsic column densities $\approx 10^{22} \mathrm{~cm}^{-2}$. For the other two galaxies with X-ray detections (40 and 56), the column density converges toward zero during the spectral fits. If we instead fix $N_{\mathrm{H}}$ to the Galactic value from the Dickey \& Lockman (1990) maps for those two sources, the best-fit $\Gamma$ values do not change significantly for either source $\left(\Gamma=1.63_{-0.25}^{+0.50}\right.$ and $1.63_{-0.47}^{+0.33}$ for galaxies 40 and 56, respectively). For consistency, in Table 3 we always quote $\Gamma$ values for the fits where $N_{\mathrm{H}}$ is allowed to vary.

The X-ray source in galaxy 111 has a high count rate, and it appears to suffer mildly from the effects of photon pileup. For sources with high count rates, two or more photons may hit a CCD detector region before the frame is read out (every $3.2 \mathrm{~s}$ for our observations), and these photons are registered as only a single event. One effect of pileup is that the observed X-ray spectrum may appear harder than the intrinsic one, because of energy migration, where the registered event has an energy equal to the sum of the multiple "piled" photons. To correct for this effect, the best-fit model parameters for galaxy 111 in Table 3 are reported using the Davis (2001) pileup model. ${ }^{11}$ The pileup fraction is $f_{\text {pile }}=0.05$ (as determined from the best-fit pileup model). The numbers of net counts and count rates reported for galaxy 111 in Table 2 are the observed numbers, without any pileup corrections; the reported fluxes and luminosities are pileup corrected (calculated within ISIS using the best-fit pileup model).

\subsection{Other $\mathrm{mBHs}$ with Chandra Coverage}

Throughout the remainder of the text, we compare our observations with 73 other mBHs with Chandra coverage. The bulk of this comparison sample includes 67 higher Eddington ratio objects, composed of 49 Chandra observations originally

\footnotetext{
$\overline{{ }^{10} \text { http://heasarc.gsfc.nasa.gov/docs/software/tools/pimms.html }}$

11 For this observation, we use a spectrum extracted from an event file that includes all events with energies $>0.3 \mathrm{keV}$ (i.e., we do not apply an $8 \mathrm{keV}$ high-energy filter); see http://cxc.harvard.edu/ciao/why/filter_energy.html.
}

published by D12, 10 from Greene \& Ho (2007c), and 8 from Desroches et al. (2009). (We refer to these as GH07 AGNs, even though 18 were originally identified as an AGN by Greene \& Ho 2004). We collectively refer to these 67 objects as the high- $L_{\mathrm{bol}} / L_{\mathrm{Edd}}$ sample. X-ray information for these 67 objects is taken directly from D12 or Desroches et al. (2009), unless stated otherwise.

The remaining six archival objects come from a search of the literature for lower Eddington ratio $\mathrm{mBHs}$ with Chandra coverage. Observations of four objects are presented by Yuan et al. (2014), whose sample includes two sources selected from the Dong et al. (2012b) catalog (SDSS J004042.10-110957.6 and SDSS J112637.74 +513423.0 ; both of which also appear in GH07), and two sources that were selected by Yuan et al. (2014) from the SDSS Data Release $^{12} 5$ (SDSS J074345.47+480813.5 and SDSS J130456.95+395529.7). These four sources span $-2.0<L_{\mathrm{bol}} / L_{\text {Edd }}<-1.3$, as calculated by Yuan et al. (2014; see their Table 1). We also consider GH07 objects with new Chandra observations presented by Gültekin et al. (2014). To avoid duplicating a similar parameter space as D12, we exclude objects from Gültekin et al. (2014) with $\log L_{\text {bol }} / L_{\text {Edd }}>-1$. Of their six sources with new Chandra observations, two remain: SDSS J121629.13+601823.5 $\left(\log L_{\mathrm{bol}} / L_{\mathrm{Edd}}=-1.3\right)$ and SDSS J132428.24+044629 $\left(\log L_{\mathrm{bol}} / L_{\mathrm{Edd}}=-1.4\right)$. Although both of these sources were initially identified as $\mathrm{mBH}$ AGNs by $\mathrm{GH} 07$, neither would have been included in our cycle-14 Chandra program because their Eddington ratios are above our $\log L_{\text {bol }} / L_{\text {Edd }}<-1.5$ criterion (plus, SDSS J121629.13+601823.5 is at too high of a redshift; $z=0.0601)$. We include them here to improve statistics, because they span a similar range in $\log L_{\text {bol }} / L_{\text {Edd }}$ as the four objects from Yuan et al. (2014). X-ray information for these combined six archival observations is taken directly from Yuan et al. (2014) and Gültekin et al. (2014), unless stated otherwise.

\subsection{X-Ray Non-detections and Stacking Analysis}

Three of our Chandra targets do not have X-ray detections, and a total of 15 other objects from our comparison sample (Section 2.3) are reported as non-detections in the literature (12 from the high- $L_{\mathrm{bol}} / L_{\mathrm{Edd}}$ sample, 2 from Yuan et al. 2014, and 1 from Gültekin et al. 2014). Because different detection thresholds are applied in each paper, we re-analyze all 15 archival observations so that we could compare upper limits on X-ray fluxes consistently between our analysis and the archival ones.

We repeat a similar data reduction on these 15 archival objects as described in Section 2.1, with the primary difference being that we use a 1." 5 aperture for extracting source counts to minimize the background in each aperture. Results of the photometry in soft $(0.5-2 \mathrm{keV})$ and hard $(2-8 \mathrm{keV})$ images are presented in Table 4, where we also repeat the analysis for our three Cycle 15 Chandra targets adopting 1." 5 apertures. In the final column of Table 4, we tabulate whether each source is considered an X-ray detection at the $95 \%$ and $99 \%$ level over the full $0.5-8 \mathrm{keV}$ band (according to the confidence interval tables in Kraft et al. 1991). We find that six objects are detected

\footnotetext{
12 Both the GH07 and Dong et al. (2012b) samples were based on SDSS Data Release 4. Yuan et al. (2014) identified these two sources by applying similar selection algorithms as Dong et al. (2012b).
} 


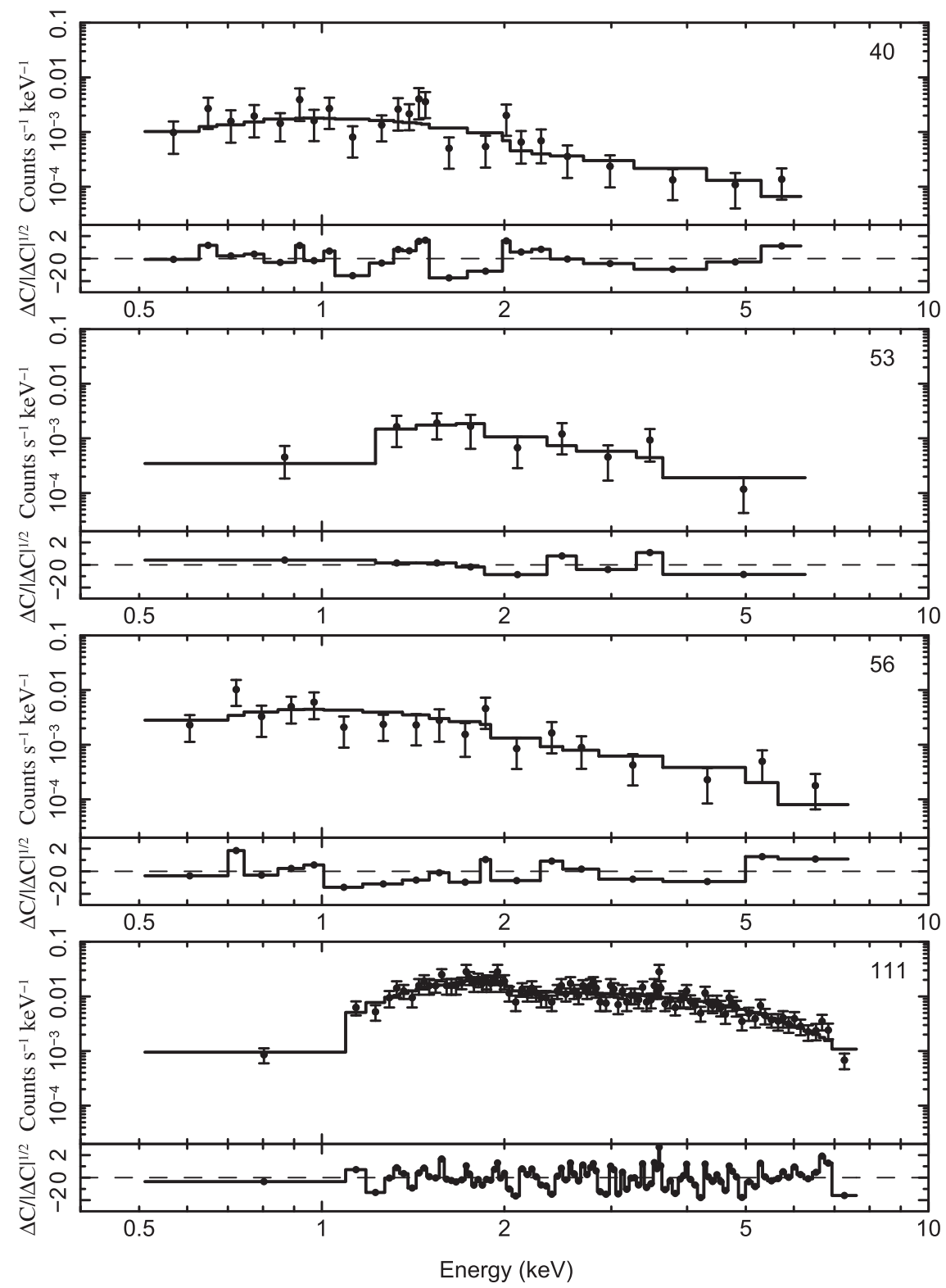

Figure 2. Spectral fits (phabs*powerlaw) to the four Chandra observations with X-ray detections (with residuals displayed as $\Delta C /|\Delta C|^{0.5}$, where $C$ is the Cash statistic). The best-fit parameters are listed in Table 3.

at the $95 \%$ level, and four are detected at the $99 \%$ level in each $0.5-8 \mathrm{keV}$ image. $^{13}$

For the six objects detected at the $95 \%$ confidence level, we adopt the fluxes quoted in Table 4 throughout the remainder of the text. The X-ray fluxes are calculated within PIMMS, assuming an absorbed power-law with $\Gamma=2.0$ and $N_{\mathrm{H}}$ set to the Galactic value from Dickey \& Lockman (1990). The fluxes are calculated over the full $0.5-8 \mathrm{keV}$ band, after applying a $92 \%$ aperture correction to account for the fraction of X-ray photons excluded by our choice of a 1 !" 5 source aperture (these aperture corrections are based on the enclosed energy fraction at $2.3 \mathrm{keV}$ at the Chandra ACIS-S3 aimpoint; including these aperture corrections produces fluxes consistent with the larger apertures we adopted in Section 2.1). For the 12 sources that

\footnotetext{
13 Our X-ray photometry on these 15 archival observations are consistent with that already reported in the literature. Differences in which are considered detections are the result of varying definitions of detection thresholds.
}

remain undetected at the $95 \%$ confidence level, we include 95\% upper limits on the full-band X-ray fluxes in Table 4.

Next, we perform a stacking analysis on the 12 sources that remain undetected at the $95 \%$ level, and on the 14 sources not detected at the $99 \%$ level. The stacked signals are presented in Table 5. For both subsets, we obtain X-ray detections in both the soft and hard energy bands at $>99 \%$ confidence. We perform the following test to confirm that the stacked detection is not an artifact of improper background estimation (see, e.g., Willott 2011; Cowie et al. 2012). For the stack of 99\% nondetections (14 objects), we blindly displace the center of each image's source extraction region by 3 ". 5 , in a randomly chosen direction for each image (the magnitude of this offset is chosen to avoid overlap with each background extraction region and the original source region). We then repeat the stacking analysis. We find only one stacked count in the soft band, and one stacked count in the hard band, which is consistent with the expected background levels of 0.6 soft and 1.1 hard counts. 
Table 3

Broadband and X-ray Spectral Properties

\begin{tabular}{lcccc}
\hline \hline GH07 ID & $\begin{array}{c}\log N_{\mathrm{H} \text { fit }} \\
\left(\mathrm{cm}^{-2}\right)\end{array}$ & $\Gamma_{\text {fit }}$ & $\alpha_{\text {ox }}$ & $\Delta \alpha_{\text {ox }}(\sigma)$ \\
$(1)$ & $(2)$ & $(3)$ & $(4)$ & $(5)$ \\
\hline 7 & $\ldots$ & $\ldots$ & $<-1.7$ & $<-0.7(-8.0)$ \\
19 & $\ldots$ & $\ldots$ & $<-1.9$ & $<-0.9(-9.4)$ \\
40 & $\ldots$ & $1.5 \pm 0.2$ & -1.4 & $-0.3(-3.6)$ \\
53 & $22.1 \pm 0.4$ & $2.3 \pm 1.5$ & -1.4 & $-0.4(-3.9)$ \\
56 & $\ldots$ & $1.6 \pm 0.4$ & -1.4 & $-0.4(-4.0)$ \\
82 & $\ldots$ & $\ldots$ & $<-1.8$ & $<-0.8(-8.5)$ \\
$111^{\mathrm{a}}$ & $22.2 \pm 0.1$ & $1.5 \pm 0.3$ & -0.8 & $0.3(2.9)$ \\
\hline
\end{tabular}

Notes. Column (1) GH07 galaxy ID. Column (2) logarithm of the best-fit column density. Blank values indicate that no spectral fit was performed (IDs 7, 19 , and 82 ), or that the best-fit column density converged toward zero (IDs 40 and 56). Column (3) best-fit photon index, $N_{E}=N_{0}\left(E / E_{0}\right)^{-\Gamma}$. Blank values indicate that no spectral fit was performed. Column (4) X-ray to UV luminosity ratio $\alpha_{\mathrm{ox}}=-0.3838 \log \left(l_{2500} / l_{2 \mathrm{keV}}\right)$, where $l_{2 \mathrm{keV}}$ and $l_{2500}$ are (unabsorbed) $\mathrm{X}$-ray and UV luminosity densities at $2 \mathrm{keV}$ and $2500 \AA$, respectively (see Section 3.2). Column (5) the difference between $\alpha_{\mathrm{ox}}$ and the value expected from the Just et al. (2007) $\alpha_{\mathrm{ox}}-l_{2500}$ relation. The statistical significance in parentheses is based on the rms scatter of $\alpha_{\mathrm{ox}}$ as a function of $l_{2500}$, as presented in Table 5 of Steffen et al. (2006). More negative numbers are "X-ray weaker."

${ }^{a}$ Spectral fit performed with the Davis (2001) pileup model, with a grade migration parameter $\alpha=0.6$. The probability of retaining $n$ events that are "piled" together is $p \sim \alpha^{n-1}$.

Finally, we use the observed hardness ratio of the stacked signals (defined by $R_{h} / R_{s}$, where $R_{h}$ and $R_{s}$ are the net count rates in the hard and soft bands, after incorporating $88 \%$ and $95 \%$ aperture corrections, respectively, that are appropriate at 1 and $4.5 \mathrm{keV}$ for $1 . " 5$ extraction regions) to explore the average spectral properties of the X-ray non-detected objects. We first assume an absorbed power-law in PIMMS, with the column density fixed to $N_{\mathrm{H}}=2 \times 10^{20} \mathrm{~cm}^{-2}$, which is the (exposuretime weighted) average of the Galactic $N_{\mathrm{H}}$ values for each source; the range of $\Gamma$ that can explain the observed hardness ratios of the stacked samples are reported in Column (10) of Table 5. We also perform a similar test fixing $\Gamma=2$ to identify the range of $N_{\mathrm{H}}$ that can replicate the observed hardness ratios (see column 11 of Table 5). The uncertainties on $\Gamma$ and $N_{\mathrm{H}}$ estimated in this manner are large, and we cannot break the degeneracy between column density and photon index with so few stacked counts. Still, we can confidently assert from this analysis that the population of X-ray non-detected $\mathrm{mBH}$ AGNs have, on average, a relatively hard observed X-ray spectrum (either caused by a photon index that is flatter than most of the X-ray detected objects, by a modest amount of intrinsic absorption, or a combination of the two).

\section{RESULTS}

\subsection{Nuclear X-Ray Emission from SMBHs}

We associate all four X-ray detections with nuclear mBHs, as described below. All four sources have hard X-ray luminosities $L_{\mathrm{X}} \gtrsim 10^{41} \mathrm{erg} \mathrm{s}^{-1}$. These X-ray luminosities are as expected given the Eddington ratios derived by GH07 for our Chandra targets: for $L_{\mathrm{bol}} / L_{\mathrm{Edd}}>10^{-2}$ and $M_{\mathrm{BH}}=10^{6} M_{\odot}$ (see Table 1), we expect $L_{\mathrm{X}}>10^{41} \mathrm{erg} \mathrm{s}^{-1}$ if $10 \%$ of the bolometric luminosity is emitted in the hard X-ray band. Excluding galaxy 111, both the soft and hard X-ray luminosities of our Chandra targets generally populate the faint end of the high- $L_{\mathrm{bol}} / L_{\mathrm{Edd}} \mathrm{GH} 07 \mathrm{mBH}$ sample (Figure 3 ). For completeness, we show the best-fit photon indices for the four X-ray detections in Figure 3(c), which cover a similar range as the high- $L_{\mathrm{bol}} / L_{\mathrm{Edd}}$ sample.

It is very unlikely that a stellar mass black hole (or even multiple stellar mass black holes within the Chandra pointspread function) could produce the observed amount of X-ray emission from each galaxy. The high-luminosity tail of a galaxy's X-ray binary (XRB) population contains the vast majority of known ultraluminous X-ray sources (ULXs; i.e., most ULXs are stellar mass black holes accreting at superEddington rates; e.g., Begelman 2002). Accounting for a combination of super-Eddington accretion and mild beaming, it is difficult for a stellar mass black hole to attain an X-ray luminosity $>10^{41} \mathrm{erg} \mathrm{s}^{-1}$ (Poutanen et al. 2007). The largest ULX catalogs observationally confirm that objects with $L_{X}>10^{41} \mathrm{erg} \mathrm{s}^{-1}$ are extremely rare and very unlikely attributed to luminous XRBs (Swartz et al. 2011; Walton et al. 2011, also see Feng \& Soria 2011 for a review). Furthermore, the brightest ULXs are typically found in galaxies with high specific star-formation rates, which contain (shortlived) high-mass XRB populations (e.g., King 2002; Grimm et al. 2003; Mineo et al. 2014). ULXs in galaxies with little star formation arise from low-mass XRBs (likely applicable to three of our four galaxies with X-ray detections, as judged from their SDSS optical colors; see Section 3.3), and they appear to always have $L_{\mathrm{X}}<2 \times 10^{39} \mathrm{erg} \mathrm{s}^{-1}$ (Irwin et al. 2004). ULXs are also $\sim 10$ times less common in such galaxies (e.g., Swartz et al. 2011; Walton et al. 2011; Plotkin et al. 2014), because the number of low-mass XRBs scales with the total stellar mass instead of the specific star-formation rate (Gilfanov 2004). Thus, the presence of hard X-rays at $>10^{41} \mathrm{erg} \mathrm{s}^{-1}$ strongly advocates that our four X-ray detected galaxies host AGN.

\subsection{X-Ray to UV Luminosity Ratios}

One of the most common ways to quantify the relative amount of accretion power outputted in the X-ray waveband is through the $\alpha_{\text {ox }}$ parameter, which measures the ratio of X-ray to UV luminosity (Tananbaum et al. 1979). We adopt $\alpha_{\mathrm{ox}}=-0.3838 \log \left(l_{2500} / l_{2 \mathrm{keV}}\right)$, where $l_{2 \mathrm{keV}}$ and $l_{2500}$ are (unabsorbed) X-ray and UV luminosity densities at rest-frame $2 \mathrm{keV}$ and $2500 \AA$, respectively. We use PIMMS and the adopted spectral parameters for each source (see Section 2.2) to calculate $l_{2 \mathrm{keV}}$. For consistency with D12, $l_{2500}$ calculations are based on broad $\mathrm{H} \alpha$ line luminosities $\left(L_{\mathrm{H} \alpha}\right)$ as follows. First, we take the GH07 $L_{\mathrm{H} \alpha}$ measurements and use the relation $L_{\mathrm{H} \alpha}=5.25 \times 10^{42}\left(L_{5100} / 10^{44} \mathrm{erg} \mathrm{s}^{-1}\right)^{1.157} \mathrm{erg} \mathrm{s}^{-1}$ (Greene \& Ho 2005) to determine the luminosity of the AGN continuum at $5100 \AA\left(L_{5100}\right)$, and we then assume that the continuum follows a power-law of the form $f_{\nu} \propto \nu^{-0.44}$ (Vanden Berk et al. 2001) to estimate $l_{2500}$. We list $\alpha_{\text {ox }}$ values in Table 3, and the $\alpha_{\text {ox }}$ distribution is shown in Figure 3(d).

Desroches et al. (2009) and D12 show that the high$L_{\text {bol }} / L_{\text {Edd }}$ sample has on average harder (i.e., less negative) $\alpha_{\mathrm{ox}}$ values compared with quasars with larger black hole masses (i.e., mBHs are systematically "X-ray brighter"). D12 explain this as being driven primarily by black hole mass, caused by lower-mass black holes being fed by higher temperature accretion disks. D12 find evidence for a weak anti-correlation between $\alpha_{\mathrm{ox}}$ and $M_{\mathrm{BH}}$ among the high- $L_{\mathrm{bol}} / L_{\mathrm{Edd}}$ sample, which supports their interpretation (an anti-correlation between $\alpha_{\mathrm{ox}}$ and $\log M_{\mathrm{BH}}$ has similarly been observed among quasars; e.g., 
Table 4

Photometry of Sources Reported as X-Ray Non-detections in the Literature

\begin{tabular}{|c|c|c|c|c|c|c|c|c|c|c|}
\hline \multirow[b]{2}{*}{$\begin{array}{l}\text { Galaxy Name } \\
\text { (SDSS J) } \\
(1)\end{array}$} & \multirow[b]{2}{*}{$\begin{array}{l}\text { ObsID } \\
\text { (2) }\end{array}$} & \multirow[b]{2}{*}{$\begin{array}{l}\tau_{\exp } \\
\text { (ks) } \\
(3)\end{array}$} & \multicolumn{2}{|c|}{ Soft $(0.5-2 \mathrm{keV})$} & \multicolumn{2}{|c|}{ Hard $(2-8 \mathrm{keV})$} & \multirow{2}{*}{$\begin{array}{l}\text { Full }(0.5-8 \mathrm{keV}) \\
\qquad \log f_{0.5-8} \\
\left(\mathrm{erg} \mathrm{s}^{-1} \mathrm{~cm}^{-2}\right) \\
(8)\end{array}$} & \multicolumn{2}{|c|}{ Detection? } & \multirow[b]{2}{*}{$\begin{array}{c}\text { References } \\
\text { (11) }\end{array}$} \\
\hline & & & $\begin{array}{c}C_{s, \text { tot }} \\
\text { (counts) } \\
\quad(4)\end{array}$ & $\begin{array}{c}B_{s} \\
\text { (counts) } \\
(5)\end{array}$ & $\begin{array}{c}C_{h, \text { tot }} \\
\text { (counts) } \\
(6)\end{array}$ & $\begin{array}{c}B_{h} \\
\text { (counts) } \\
(7)\end{array}$ & & $\begin{array}{l}95 \% \\
(9)\end{array}$ & $\begin{array}{l}99 \% \\
(10)\end{array}$ & \\
\hline $003552.26+011249.4$ & 15015 & 15.9 & 0 & 0.06 & 0 & 0.09 & $<-14.8$ & $\mathrm{~N}$ & $\mathrm{~N}$ & this work \\
\hline $004042.10-110957.6$ & 9235 & 4.7 & 1 & 0.02 & 1 & 0.1 & $<-14.1$ & $\mathrm{~N}$ & $\mathrm{~N}$ & Y14 \\
\hline $023310.79-074813.3$ & 15017 & 10.0 & 0 & 0.06 & 0 & 0.06 & $<-14.6$ & $\mathrm{~N}$ & $\mathrm{~N}$ & this work \\
\hline $094310.12+604559.1$ & 5661 & 5.0 & 2 & 0.07 & 0 & 0.1 & $<-14.0$ & $\mathrm{~N}$ & $\mathrm{~N}$ & GH07c \\
\hline $095306.81+365028.0$ & 15020 & 20.9 & 0 & 0.1 & 0 & 0.2 & $<-15.0$ & $\mathrm{~N}$ & $\mathrm{~N}$ & this work \\
\hline $095330.53+562653.4$ & 11452 & 2.0 & 3 & 0.01 & 0 & 0.01 & $-14.0_{-0.5}^{+0.4}$ & $\mathrm{Y}$ & $\mathrm{N}$ & D12 \\
\hline $105755.66+482502.0$ & 11455 & 2.0 & 1 & 0.01 & 0 & 0.02 & $<-13.8$ & $\mathrm{~N}$ & $\mathrm{~N}$ & D12 \\
\hline $112637.74+513423.0$ & 9234 & 4.7 & 1 & 0.05 & 0 & 0.02 & $<-14.2$ & $\mathrm{~N}$ & $\mathrm{~N}$ & Y14 \\
\hline $114343.76+550019.3$ & 11460 & 1.8 & 0 & 0.0 & 0 & 0.01 & $<-13.9$ & $\mathrm{~N}$ & $\mathrm{~N}$ & D12 \\
\hline $115138.24+004946.4$ & 7735 & 4.7 & 0 & 0.03 & 1 & 0.09 & $<-14.2$ & $\mathrm{~N}$ & $\mathrm{~N}$ & D09 \\
\hline $121629.13+601823.5$ & 13860 & 24.0 & 21 & 0.09 & 5 & 0.2 & $-14.1_{-0.2}^{+0.1}$ & Y & $\mathrm{Y}$ & G14 \\
\hline $131926.52+105610.9$ & 11470 & 1.8 & 5 & 0.03 & 2 & 0.0 & $-13.6_{-0.3}^{+0.3}$ & $\mathrm{Y}$ & $\mathrm{Y}$ & D12 \\
\hline $144052.60-023506.2$ & 11474 & 1.8 & 0 & 0.03 & 1 & 0.07 & $<-13.7$ & $\mathrm{~N}$ & $\mathrm{~N}$ & D12 \\
\hline $144507.30+593649.9$ & 7738 & 4.7 & 2 & 0.02 & 6 & 0.1 & $-13.9_{-0.3}^{+0.2}$ & Y & Y & D09 \\
\hline $153656.44+312248.1$ & 11476 & 1.8 & 0 & 0.02 & 1 & 0.05 & $<-13.7$ & $\mathrm{~N}$ & $\mathrm{~N}$ & D12 \\
\hline $163159.59+243740.2$ & 11483 & 1.9 & 24 & 0.05 & 8 & 0.02 & $-12.9_{-0.2}^{+0.1}$ & Y & $\mathrm{Y}$ & D12 \\
\hline $170246.09+602818.9$ & 7739 & 4.7 & 0 & 0.03 & 2 & 0.1 & $<-14.0$ & $\mathrm{~N}$ & $\mathrm{~N}$ & D09 \\
\hline $233837.10-002810.3$ & 5667 & 4.7 & 2 & 0.1 & 2 & 0.1 & $-14.2_{-0.5}^{+0.3}$ & Y & $\mathrm{N}$ & GH07c \\
\hline
\end{tabular}

Note. Column (1) galaxy name. Column (2) Chandra observation ID. Column (3) effective exposure time of Chandra observation. Column (4) total number of soft $(0.5-2 \mathrm{keV}) \mathrm{X}$-ray counts within a 1 .' 5 circular aperture centered on the optical source position (no background subtraction). Column (5) expected number of soft $(0.5-2 \mathrm{keV})$ background counts within each source aperture, estimated from background annuli with inner and outer radii of $5^{\prime \prime}$ and $10^{\prime \prime}$, respectively. Column (6) total number of hard (2-8 keV) X-ray counts. Column (7) expected number of hard (2-8 keV) background counts. Column (8) logarithm of the X-ray flux over the full $0.5-8 \mathrm{keV}$ band, assuming an absorbed power-law in PIMMS, with $\Gamma=2.0$ and $N_{\mathrm{H}}$ set to the Galactic value along the line of sight. A $92 \%$ aperture correction (calculated at $2.3 \mathrm{keV}$ ) is applied to the net count rates for the flux calculation to account for the fraction of source photons missed by our choice of 1 !" 5 aperture. If a source is not detected within the $0.5-8 \mathrm{keV}$ band, then flux upper limits are reported at the $95 \%$ confidence level. Column (9) flag denoting whether an X-ray source is detected (Y) or not detected (N) at the 95\% confidence level over the full 0.5-8 keV band (according to Kraft et al. 1991). Column (10) same as column (9), but at the 99\% confidence level. Column (11) reference of paper that originally published each Chandra observation. D09, Desroches et al. (2009); D12, Dong et al. (2012a); G14, Gültekin et al. (2014); GH07c, Greene \& Ho (2007c); Y14, Yuan et al. (2014).

Kelly et al. 2008). However, D12 also show that $\alpha_{\text {ox }}$ is not as hard as expected, if one were to extrapolate from the wellknown anti-correlation between $\alpha_{\mathrm{ox}}$ and $l_{2500}$ that is defined by more luminous (and massive) quasars (e.g., Avni \& Tananbaum 1982; Steffen et al. 2006; Just et al. 2007). A potential flattening of the $\alpha_{\mathrm{ox}}-l_{2500}$ relation at low luminosities was also hinted at by Steffen et al. (2006), and also seen by Maoz (2007) for a sample of 13 low ionization nuclear emission line region galaxies.

While most of our Chandra targets are also X-ray brighter than luminous quasars, they tend to be X-ray weaker (on average) than expected for their UV luminosities-excluding galaxy 111, the six other targets have $\Delta \alpha_{\mathrm{ox}}=\alpha_{\mathrm{ox}}-\alpha_{\mathrm{ox}, \exp }<-0.3$ (see Table 3), where $\alpha_{\mathrm{ox}, \exp }$ is the average $\alpha_{\text {ox }}$ expected from the Just et al. (2007) $\alpha_{\text {ox }}-l_{2500}$ relation. Our targets' $\Delta \alpha_{\mathrm{ox}}$ values correspond to being X-ray weaker than $\alpha_{\text {ox, exp }}$ at the 3.6-8.5 $\sigma$ level (see Table 3; the rms $\sigma$ deviation for each object is tabulated from Steffen et al. 2006). Finally, we note that galaxy 111 is the $\mathrm{X}$-ray brightest $\mathrm{mBH}$ from GH07 observed so far, but it does not have an unusual X-ray spectrum or column density that would indicate that it is fundamentally different than the other GH07 objects.

\subsection{Narrow Line Emission and AGN Classification}

All seven Chandra targets are formally below the broad-line detection thresholds of $\mathrm{GH} 07$, who primarily require $f_{\mathrm{H} \alpha} / \sigma_{\mathrm{rms}}>200$ and $\mathrm{EW}[\mathrm{H} \alpha]>15 \AA\left(f_{\mathrm{H} \alpha}\right.$ is the $\mathrm{H} \alpha$ line flux, $\sigma_{\text {rms }}$ is the rms deviation of the continuum subtracted spectrum, and $\mathrm{EW}[\mathrm{H} \alpha]$ is the $\mathrm{H} \alpha$ equivalent width; see Greene \& Ho 2007b for details). Our targets were included in the GH07 sample because visual inspection of their spectra (by $\mathrm{GH} 07$ ) revealed broad and interesting $\mathrm{H} \alpha$, and they were subsequently flagged by GH07 as less secure "candidate" black holes (which they term as their $c$ sample). We assess our targets' AGN identifications here by re-examining their optical properties in conjunction with our new Chandra X-ray constraints.

As an initial cross-check, we consult the results of an independent optical analysis by Reines \& Volonteri (2015), who analyzed the SDSS spectra of $\sim 67,000$ emission line galaxies. Reines \& Volonteri (2015) detected broad $\mathrm{H} \alpha$ in all our targets except for galaxy $7 .{ }^{14}$ We therefore operate under the assumption that only for galaxy 7 could the broad $\mathrm{H} \alpha$ seen by GH07 during visual inspection be a statistical "false positive." That is, we consider the claim for the presence of broad $\mathrm{H} \alpha$ in these objects to be robust. We stress, however, that broad $\mathrm{H} \alpha$ on its own does not prove that an AGN is present, especially in star-forming galaxies where there could be sources of contamination from young stars and/or supernovae (see e.g., Filippenko 1989; Greene \& Ho 2004; Reines et al. 2013, Baldassare et al. 2016).

\footnotetext{
${ }^{14}$ Note that not all our targets are included in the final 262-object AGN sample of Reines \& Volonteri (2015), as they require both broad $\mathrm{H} \alpha$ and Seyfert-like narrow emission lines.
} 
Table 5

X-Ray Stacking Analysis

\begin{tabular}{|c|c|c|c|c|c|c|c|c|c|c|}
\hline \multirow[b]{2}{*}{ Stack } & \multirow[b]{2}{*}{$N_{\mathrm{src}}$} & \multirow[b]{2}{*}{$\begin{array}{c}\tau_{\text {tot }} \\
(\mathrm{kss})\end{array}$} & \multicolumn{3}{|c|}{ Soft $(0.5-2 \mathrm{keV})$} & \multicolumn{3}{|c|}{ Hard $(2-8 \mathrm{keV})$} & \multirow[b]{2}{*}{$\Gamma_{\text {fixed } N_{\mathrm{H}}}$} & \multirow[b]{2}{*}{$\begin{array}{c}\log N_{\mathrm{H}_{\text {fixed }}} \\
\left(\mathrm{cm}^{-2}\right) \\
(11)\end{array}$} \\
\hline & & & $\begin{array}{c}C_{s, \text { tot }} \\
\text { (counts) } \\
\text { (4) }\end{array}$ & $\begin{array}{c}B_{s} \\
\text { (counts) } \\
(5)\end{array}$ & $\begin{array}{c}R_{S} \\
\left(\text { counts s}^{-1}\right) \\
(6)\end{array}$ & $\begin{array}{c}C_{h, \text { tot }} \\
\text { (counts) } \\
\text { (7) }\end{array}$ & $\begin{array}{c}B_{h} \\
\text { (counts) } \\
(8)\end{array}$ & $\begin{array}{c}R_{h} \\
\text { (counts s }^{-1} \text { ) } \\
(9)\end{array}$ & & \\
\hline $95 \%$ conf. & 12 & 77.9 & 5 & 0.5 & $6.1 \times 10^{-5}$ & 6 & 1.0 & $7.3 \times 10^{-5}$ & $0.6_{-0.6}^{+1.6}$ & $22.1(<22.3)$ \\
\hline $99 \%$ conf. & 14 & 84.6 & 10 & 0.6 & $1.1 \times 10^{-4}$ & 8 & 1.1 & $9.3 \times 10^{-5}$ & $1.0_{-0.5}^{+1.1}$ & $21.8(<22.1)$ \\
\hline
\end{tabular}

Note. Column (1) subset of objects included in stacking analysis (non-detections at either the $95 \%$ or $99 \%$ confidence level). Column (2) number of objects included. Column (3) total stacked exposure time. Column (4) total number of stacked soft $(0.5-2 \mathrm{keV}) \mathrm{X}$-ray counts within a 1". 5 circular aperture centered on the optical source position (no background subtraction). Column (5) expected number of stacked soft (0.5-2 keV) background counts within each source aperture. Column (6) stacked net soft count rate $(0.5-2 \mathrm{keV})$. A $95 \%$ aperture correction (calculated at $1 \mathrm{keV}$ ) is applied to account for the fraction of source photons missed by our choice of 1" 5 aperture. Column (7) total number of stacked hard (2-8 keV) X-ray counts. Column (8) expected number of stacked hard (2-8 keV) background counts within each source aperture. Column (9) stacked net hard count rate (2-8 keV). An $88 \%$ aperture correction (calculated at $4.5 \mathrm{keV}$ ) is applied to account for the fraction of source photons missed by our choice of 1". 5 aperture. Column (10) estimated $\Gamma$ that can explain the stacked hardness ratio from the stacked signals, assuming an absorbed power-law, and keeping the column density frozen at $N_{\mathrm{H}}=2 \times 10^{20} \mathrm{~cm}^{-2}$ (the average Galactic column density along each line of sight, weighted by the exposure time of each observation). Column (11) estimated $\log N_{\mathrm{H}}$ that can explain the stacked hardness ratios, assuming an absorbed power-law with $\Gamma=2$. The lower-limit on the $90 \%$ confidence interval is unconstrained by the data, so in lieu of error bars, we report the $90 \%$ confidence upper limit in parentheses for this column.

In Figure 4 we show the location of each of our targets in the Baldwin-Phillips-Terlevich (BPT) diagram (e.g., Baldwin et al. 1981; Kewley et al. 2001, 2006; Kauffmann et al. 2003), which uses narrow emission line diagnostics to separate purely star-forming galaxies from galaxies with harder (AGNlike) ionizing continua. For reference, we include the high$L_{\text {bol }} / L_{\text {Edd }}$ sample in Figure 4 . Three of our Chandra targets have the narrow optical line ratios typical of Seyfert galaxies (galaxies 19, 40, and 111). These three galaxies also appear to have red SDSS optical colors (see bottom panels of Figure 4), which suggests negligible star formation (although, see Jiang et al. 2011 for a high spatial resolution morphological study that indicates all three galaxies contain a disk component). The combination of the narrow-line diagnostics and the presence of broad $\mathrm{H} \alpha$ emission highly suggests that all three of these galaxies host an AGN. Galaxies 40 and 111 also display X-ray emission that confirms their AGN nature (see Section 3.1). We note that the absence of X-ray emission is not sufficient to exclude the presence of an AGN. Although we do not detect $\mathrm{X}$-rays from galaxy 19 , we have a sensitive limit $\left(\alpha_{\text {ox }}<-1.72\right)$ that places it in the X-ray weak tail defined by the high- $L_{\text {bol }} / L_{\text {Edd }}$ sample (D12, also see Section 4 ).

None of the other four galaxies show obvious optical narrow-line AGN signatures in Figure 4. However, two are still very likely AGNs based on their X-ray properties (galaxies 53 and 56; we note that Dong et al. 2012b independently classify galaxy 53 as an mBH AGN from broad $\mathrm{H} \alpha$ in its optical SDSS spectrum). Galaxies 7 and 82 do not show X-ray emission or AGN-like narrow-line ratios and have blue SDSS optical colors; it is unclear whether galaxy 7 displays broad $\mathrm{H} \alpha$. We are therefore uncertain of the AGN nature for galaxies 7 and 82, and here consider them to be low-confidence AGN candidates. We note that galaxy 7 is not included in the low$M_{\mathrm{BH}}$ sample of Dong et al. (2012b), but galaxy 82 is.

Among the six mBHs with low- $L_{\mathrm{bol}} / L_{\mathrm{Edd}}$ for which we take $\mathrm{X}$-ray information from the literature, the two from Gültekin et al. (2014) were also flagged by GH07 as part of their $c$ sample. Following similar arguments as above, we consider both sources to be AGNs here: both have AGN-like log [O III]/ $\mathrm{H} \beta>1.3$ measured by GH07 and nuclear X-ray point sources. Yuan et al. (2014) discuss classification of their four targets in detail, and we consider all four of their objects to be bona fide $\mathrm{mBH}$ AGN.

In summary, we consider galaxies 40 and 111 to be AGNs based on their optical and X-ray properties, and galaxy 19 to be an X-ray weak AGN. Galaxies 53 and 56 appear to be AGNs that lack AGN-like narrow emission lines, and we consider galaxies 7 and 82 to be (lower-confidence) AGN candidates.

\subsection{Bolometric Luminosities and Eddington Ratios}

Throughout the text, we generally adopt the Eddington ratios calculated by $\mathrm{GH} 07$ for our Chandra targets. For these calculations, a bolometric correction of $L_{\text {bol }}=9.8 L_{5100}$ (McLure \& Dunlop 2004) is adopted, where $L_{5100}$ is the luminosity of the AGN continuum at $5100 \AA$ (which is estimated from the broad $\mathrm{H} \alpha$ line luminosity; see Section 3.2). We adopt the GH07 $L_{\text {bol }} / L_{\text {Edd }}$ estimates to ease comparison with the literature, as $L_{\mathrm{bol}} / L_{\mathrm{Edd}}$ estimates for the high $-L_{\mathrm{bol}} / L_{\mathrm{Edd}}$ sample are also drawn from GH07. It is possible that this specific choice of bolometric correction could systematically over- or underestimate $L_{\text {bol }} / L_{\text {Edd }}$. However, such a bias would affect the the entire sample in the same direction, on average. So, by calculating $L_{\text {bol }} / L_{\text {Edd }}$ in a consistent manner between all subsets, we can reliably search for trends as a function of (relative) Eddington ratio, as long as we bear in mind the possibility of a systematic offset for the entire sample when interpreting the results.

At the moment, bolometric corrections for $\mathrm{mBH}$ AGNs are still poorly constrained, and empirical constraints require multiwavelength, high spatial resolution imaging of the nuclear emission for a sizable sample of objects. In the meantime, we explore three other methods of applying bolometric corrections to estimate $L_{\text {bol }}$ for AGNs, which are described below and summarized in Table 6:

1. $k\left(M_{\mathrm{BH}}\right)=L_{\mathrm{bol}} / L_{5100}$ : D12 suggest that the bolometric correction could include a black hole mass term, such that $\log \left(L_{\mathrm{bol}} / L_{5100}\right)=-0.54 \log M_{\mathrm{BH}}+5.43 \quad$ (see their Equation (3)). For a $10^{6} M_{\odot} \mathrm{mBH}$, this leads to $L_{\text {bol }}=155 L_{5100}$, which increases the estimates from GH07 by $\approx 1.2$ dex (see column 3 of Table 6 ).

2. $k_{1}=L_{\mathrm{bol}} / L_{5100}$ : we also estimate $L_{\mathrm{bol}} / L_{\mathrm{Edd}}$ from the strength of [O III] line emission in the SDSS optical 

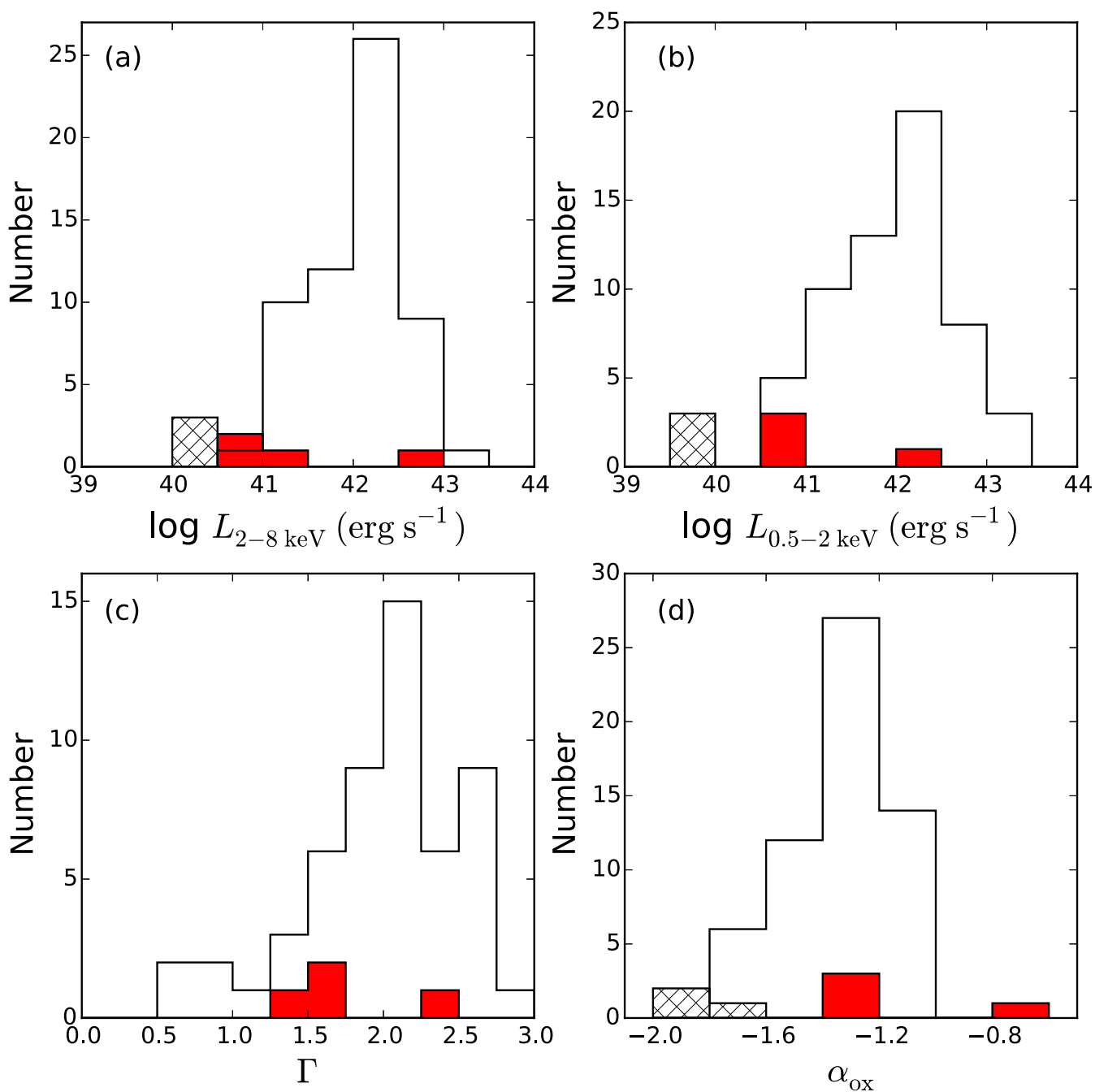

Figure 3. X-ray properties of our seven lower Eddington ratio Chandra targets (red histograms), with cross-hatched histograms indicating upper limits. The X-ray properties of the high- $L_{\mathrm{bol}} / L_{\mathrm{Edd}}$ sample are shown for reference (open histograms). For clarity, only high- $L_{\mathrm{bol}} / L_{\mathrm{Edd}}$ objects with X-ray detections are included. (a) Hard X-ray luminosity from 2 to $8 \mathrm{keV}$. (b) Soft X-ray luminosity from 0.5 to $2 \mathrm{keV}$. (c) Best-fit photon spectral index $\Gamma$ (only four Chandra targets with X-ray detections are shown). (d) X-ray to UV luminosity ratio parameterized by $\alpha_{\mathrm{ox}}$. More negative numbers are "X-ray weaker."

spectra. Following Kauffmann \& Heckman (2009), we assume $L_{\text {bol }} \approx 500-800 L[\mathrm{O}$ III $]$. When calculating $L$ [0 III], we include a correction for extinction by estimating $E(B-V)$ from the Balmer-decrement in the SDSS spectra (assuming a Cardelli et al. 1989 extinction curve and $L_{\mathrm{H} \alpha} / L_{\mathrm{H} \beta}=3.1$, which is typically adopted for AGNs to include photoionization + collisional enhancement of Hydrogen lines; e.g., Halpern \& Steiner 1983). Because this $L_{\text {bol }}$ estimate assumes photoionization by an AGN continuum, we only perform the calculation for the three targets with AGN-like narrow emission line ratios in Figure 4 (galaxies 19, 40, 111; see column 4 of Table 6).

3. $k_{2}=L_{\mathrm{bol}} / L_{2-10 \mathrm{keV}}$ : we also estimate $L_{\mathrm{bol}} / L_{\mathrm{Edd}}$ from the observed X-ray luminosities. Following Yuan et al. (2014), (see their Section 5), we apply an X-ray bolometric correction for $\mathrm{mBHs}$ of $L_{\mathrm{bol}}=7-20 L_{2-10 \mathrm{keV}}$ (see column 5 of Table 6).

Both the mass-dependent and $L[\mathrm{O} \mathrm{III}]$-based bolometric corrections suggest more rapidly accreting AGNs than estimated by GH07. The X-ray based bolometric corrections generally predict lower Eddington ratios (except for galaxy 111, the lone object with a positive $\Delta \alpha_{\text {ox }}$ value). The discrepancy between different methods of calculating $L_{\text {bol }} / L_{\text {Edd }}$ serves as a quantitative guide for the degree to which systematics may affect our adopted $\log L_{\text {bol }} / L_{\text {Edd }}$ estimates. We stress, however, that the discrepancies between different $L_{\text {bol }}$ estimates do not imply that the GH07 values must be incorrect or biased. Rather, the discrepancies motivate the need for high-resolution broadband imaging of the nuclei of a large sample of $\mathrm{mBH} \mathrm{AGN}$, to provide observational constraints to calibrate bolometric corrections against.

\section{DISCUSSION}

Here, we compare the X-ray properties of the lower Eddington ratio AGNs to the high- $L_{\text {bol }} / L_{\text {Edd }}$ sample (differences between the X-ray properties of accreting mBHs versus SMBHs have already been explored in depth by D12; also see Greene \& Ho 2007c; Desroches et al. 2009). We exclude the two lower-confidence AGN candidates (galaxies 7 and 82) from the following discussion, unless stated otherwise. Our low- $L_{\text {bol }} / L_{\text {Edd }}$ sample therefore contains 11 objects, and the high- $L_{\text {bol }} / L_{\text {Edd }}$ comparison samples contains 67 objects. As noted in Section 3.4, we adopt the $L_{\text {bol }} / L_{\text {Edd }}$ estimates from $\mathrm{GH} 07$ because that provides the most straightfoward 

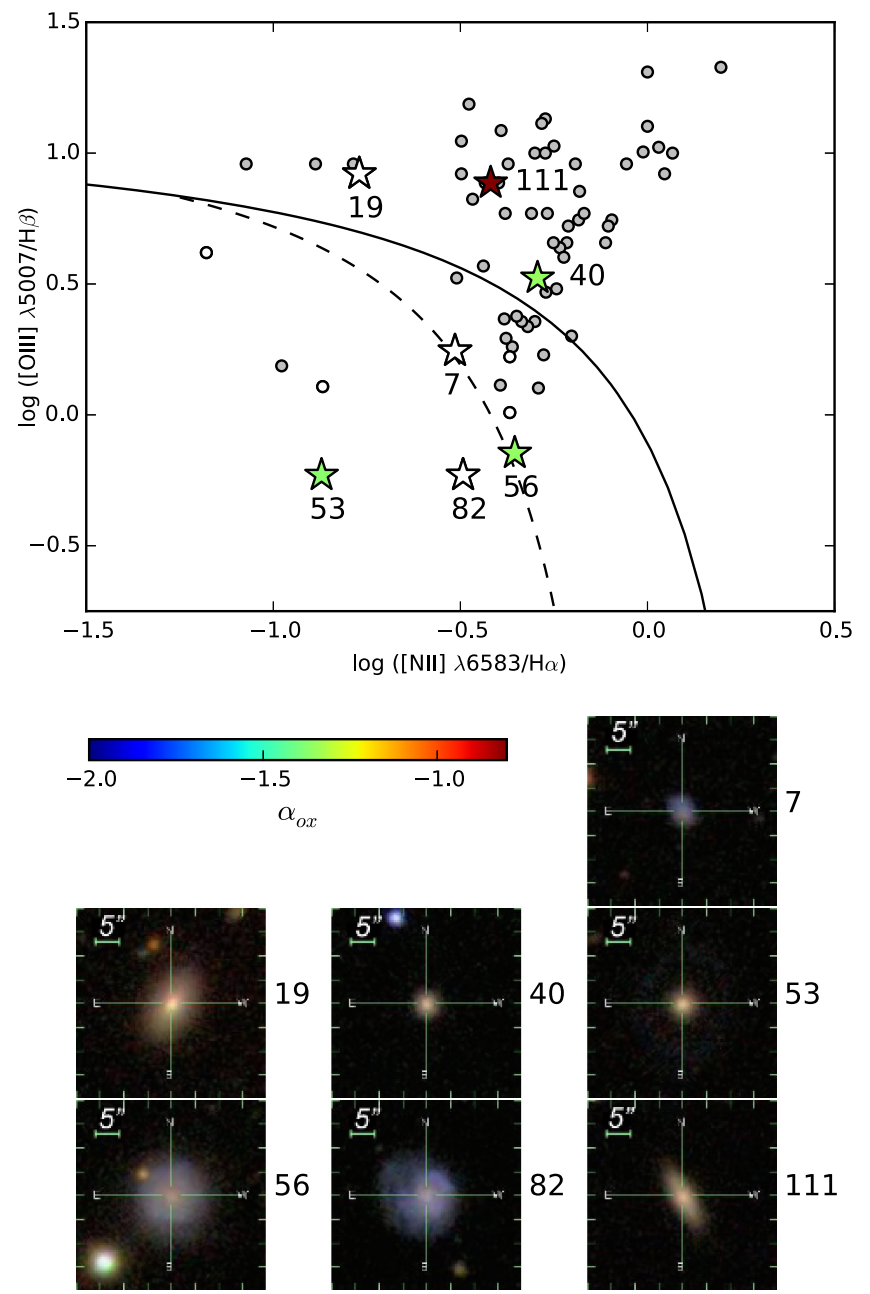

Figure 4. Top panel: BPT diagram showing narrow emission line ratios for our seven Chandra X-ray targets (star symbols), color-coded by X-ray brightness $\alpha_{\text {ox }}$ (see color bar). Open star symbols denote X-ray non-detections. The solid curve shows the "maximum starburst line" from Kewley et al. (2006), derived from pure stellar photoionization models. Galaxies above the solid curve are "Seyfert-like." The dashed curve shows the empirical dividing line between star-forming and active galaxies (Kauffmann et al. 2003). AGNs from the high$L_{\text {bol }} / L_{\text {Edd }}$ sample are overplotted for comparison (circles), with open symbols denoting X-ray non-detections. The bottom panels show SDSS gri color composite images of each of our Chandra targets. The green scale bar at the top left of each image represents $5^{\prime \prime}$.

method for uniformly comparing $L_{\mathrm{bol}} / L_{\mathrm{Edd}}$ across the entire 78-object sample. While these estimates may be systematically offset from the "true" $L_{\text {bol }} / L_{\text {Edd }}$ values, they provide a reliable tracer for the relative Eddington ratios between objects in the full sample. We cannot calculate $L_{\text {bol }} / L_{\text {Edd }}$ from $L[\mathrm{O}$ III $]$ across the entire sample because not all objects show narrow-line AGN signatures. The full 78-object sample shows a large dispersion in $\alpha_{\text {ox }}$ (see Section 4.1), which implies that applying an X-ray based bolometric correction for determining $L_{\mathrm{bol}} / L_{\mathrm{Edd}}$ is not straightforward, and would likely require an undetermined correction that is dependant on $\alpha_{\text {ox }}$.

D12 report on the lack of a correlation between $\alpha_{\text {ox }}$ and $\log L_{\text {bol }} / L_{\text {Edd }}$ within just the high- $L_{\text {bol }} / L_{\text {Edd }}$ sample (also see Greene \& Ho 2007c; Desroches et al. 2009). After extending the dynamic range in $\log L_{\mathrm{bol}} / L_{\mathrm{Edd}}$ by almost an order of magnitude, we also do not see a correlation (Figure 5). We perform a linear regression on $\alpha_{\mathrm{ox}}$ versus $\log L_{\mathrm{bol}} / L_{\mathrm{Edd}}$ (including upper limits on $\alpha_{\text {ox }}$; Kelly 2007) and find a slope
Table 6

$\log L_{\text {bol }} / L_{\text {Edd }}$ Using Different Bolometric Corrections

\begin{tabular}{lcccc}
\hline \hline \multirow{2}{*}{$\begin{array}{l}\text { GH07 ID } \\
(1)\end{array}$} & $\begin{array}{c}9.8 L_{5100} \\
(2)\end{array}$ & $k\left(M_{\mathrm{BH}}\right) L_{5100}$ & $k_{1} L[\mathrm{O}$ III] & $k_{2} L_{2-10 \mathrm{keV}}$ \\
\cline { 2 - 5 } & -1.7 & -0.4 & $(4)$ & $<L_{\mathrm{bol}} / L_{\mathrm{Edd}}$ \\
\hline 7 & -1.6 & -0.5 & -0.8 to -0.6 & $<-2.6$ \\
19 & -1.5 & -0.3 & -1.1 to -0.8 & -2.1 to -1.6 \\
40 & -1.8 & -0.7 & $\ldots$ & -2.6 to -2.2 \\
53 & -2.0 & -0.9 & $\ldots$ & -2.5 to -2.1 \\
56 & -1.6 & -0.4 & $\ldots$ & $<-2.4$ \\
82 & -1.5 & -0.4 & -0.5 to -0.3 & -0.5 to -0.0 \\
111 & & & & $\ldots$
\end{tabular}

Note. Column (1) GH07 galaxy ID. Column (2) $\log L_{\mathrm{bol}} / L_{\mathrm{Edd}}$ from GH07 (repeated from Table 1), assuming $L_{\mathrm{bol}}=9.8 L_{5100}$. Column (3) $\log L_{\mathrm{bol}} / L_{\mathrm{Edd}}$ assuming $L_{\mathrm{bol}}=10^{5.43} M_{\mathrm{BH}}^{-0.54} L_{5100} \quad$ (see D12). Column (4) range of $\log L_{\mathrm{bol}} / L_{\mathrm{Edd}}$, assuming $L_{\mathrm{bol}}=k_{1} L[\mathrm{O} \mathrm{III}]$, where $k_{1}=500-800$ (see Kauffmann \& Heckman 2009). $L[\mathrm{O}$ III] is calculated by applying a Balmer-decrement based correction for extinction to the [O III] line fluxes presented by GH07. Values are presented only for our three Chandra targets displaying narrow-line emission indicative of photoionization by an AGN. Column (5) range (or limits) of $\log L_{\mathrm{bol}} / L_{\mathrm{Edd}}$ assuming $L_{\mathrm{bol}}=k_{2} L_{2-10 \mathrm{keV}}$, where $k_{2}=7-20$, and $L_{2-10 \mathrm{keV}}$ is the X-ray luminosity (or limit) determined by our Chandra observations.

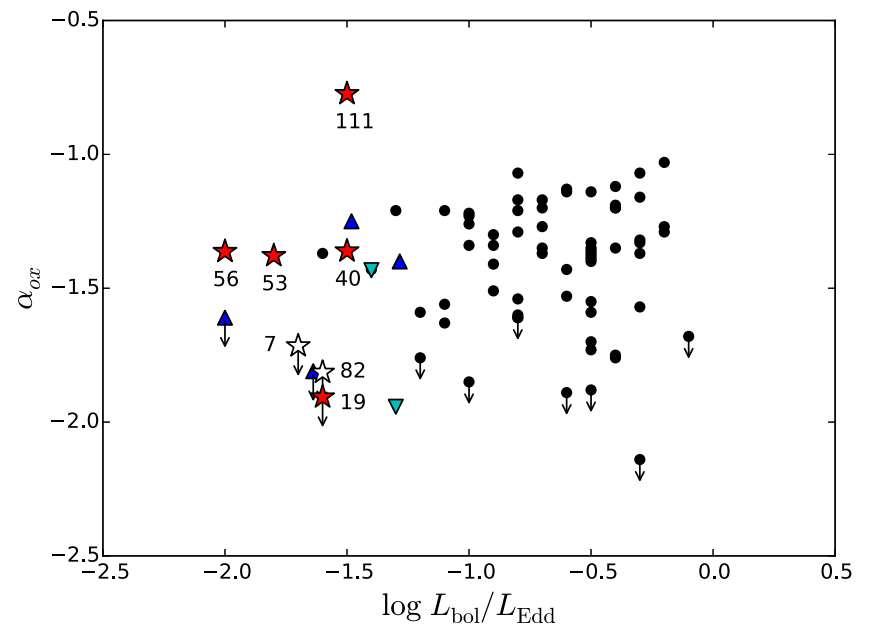

Figure 5. $\alpha_{\mathrm{ox}}$ as a function of Eddington ratio for our Chandra targets (red filled star symbols), the four mBH AGNs with $\log L_{\text {bol }} / L_{\text {Edd }}<-1$ from Yuan et al. (2014; blue triangles), the two $\mathrm{mBH}$ AGNs with $\log L_{\text {bol }} / L_{\text {Edd }}<-1$ from Gültekin et al. (2014; cyan upside-down triangles), and the high- $L_{\text {bol }} / L_{\text {Edd }}$ sample (circles). Arrows denote upper limits on $\alpha_{\text {ox }}$. The open star symbols represent our two less-confident AGN candidates (galaxies 7 and 82; see Section 3.3).

consistent with zero $(0.1 \pm 0.1)$. Furthermore, generalized Kendall's $\tau$ and Spearman's $\rho$ correlation tests on the combined 78-object sample both indicate that no correlation is present at the $p=0.1$ level. We incorporate upper limits on the X-ray non-detections when running these correlation tests by using the Astronomy SURVival Analysis (ASURV) package rev 1.2 (Lavalley et al. 1992), which implements the statistical methods presented in Feigelson \& Nelson (1985). The lack of a correlation within just the 67 -object high- $L_{\mathrm{bol}} / L_{\mathrm{Edd}}$ sample reported by D12 is therefore not solely due to their limited dynamic range.

In addition, we do not see any evidence for statistically different X-ray properties between the low- and high- $L_{\text {bol }} / L_{\text {Edd }}$ samples. A Peto-Prentice test (run through ASURV to 
incorporate upper limits) indicates that the low and high Eddington ratio samples do not follow statistically different distributions in $\alpha_{\mathrm{ox}}(p=0.2$; also see Figures 3(d) and 5). We also estimate the average $\alpha_{\text {ox }}$ values for each distribution using the Kaplan-Meier estimator in ASURV. The 11 lower$L_{\text {bol }} / L_{\text {Edd }}$ AGNs have a mean $\left\langle\alpha_{\text {ox }}\right\rangle=-1.5 \pm 0.1$, which is comparable to $\left\langle\alpha_{\text {ox }}\right\rangle=-1.4 \pm 0.04$ for the 67-object high$L_{\text {bol }} / L_{\text {Edd }}$ sample. Furthermore, for our four Chandra objects to which we could fit a spectrum, we also do not see any meaningful differences in the spectral properties between the two samples (Figure 3(c)). We may have expected to see a correlation between (hard X-ray) $\Gamma$ and $\log L_{\text {bol }} / L_{\mathrm{Edd}}$, as is observed for luminous quasars with $\log L_{\text {bol }} / L_{\text {Edd }} \gtrsim-2$ (e.g., Shemmer et al. 2008; Risaliti et al. 2009; Brightman et al. 2013; Ricci et al. 2013), but proper investigation will require a sample with tighter X-ray spectral constraints, and thus deeper X-ray observations.

\subsection{The X-Ray Weak Tail}

The lower- $L_{\text {bol }} / L_{\text {Edd }}$ mBHs appear to show as wide of a spread in $\alpha_{\text {ox }}$ as the high- $L_{\text {bol }} / L_{\text {Edd }}$ sample (see Figure 5). Given that very few $\mathrm{mBHs}$ display signs of X-ray absorption (and those that do typically have moderate column densities, $N_{\mathrm{H}} \approx 10^{22} \mathrm{~cm}^{-2}$ ), some mBHs might be intrinsically X-ray weak. They could be similar to the nearby $(z=0.192)$ narrowline Seyfert 1 galaxy PHL 1811, which may have a smaller or quenched X-ray corona (e.g., Leighly et al. 2007a, 2007b). Intriguingly, the range in $\alpha_{\mathrm{ox}}$ displayed by the $\mathrm{mBHs}$ is also reminiscent of the X-ray properties of weak emission line quasars (WLQs; see, e.g., Shemmer et al. 2009; Wu et al. 2011, 2012; Luo et al. 2015). WLQs are higher-redshift (mostly known at $z \gtrsim 1$ ), unobscured, radio-quiet quasars that have unusually weak high-ionization broad emission lines (especially Ly $\alpha$ and C IV $\lambda 1549$; see, e.g., Fan et al. 1999; Diamond-Stanic et al. 2009; Plotkin et al. 2010a, 2010b; Shemmer et al. 2010; Lane et al. 2011). Approximately 50\% of known WLQs are significantly X-ray weaker than expected for their UV luminosities (with $\Delta \alpha_{\mathrm{ox}}<-0.2$; see Shemmer et al. 2009; Wu et al. 2012; Luo et al. 2015).

In Figure 6, we compare the $\alpha_{\mathrm{ox}}$ distribution of mBHs to WLQs as a function of $l_{2500}$, with typical SDSS Type-1 quasars from Just et al. (2007) plotted for reference. We include 38 WLQs that were optically selected from the SDSS, which have $z>1.5$ (to ensure SDSS spectroscopic coverage of the high-ionization emission line $\mathrm{C}$ IV) and Chandra X-ray observations presented by $\mathrm{Wu}$ et al. $(2011,2012)$ and Luo et al. (2015). ${ }^{15}$ The lack of WLQs at $l_{2500}<10^{31} \mathrm{erg} \mathrm{s}^{-1} \mathrm{~Hz}^{-1}$ in Figure 6 is largely (but unlikely solely) due to the restriction in redshift.

It is clear from Figure 6 that both the $\mathrm{mBH}$ and WLQ populations display a larger dispersion in $\alpha_{\text {ox }}$ compared with "normal" Type-1 SDSS quasars. To quantify the dispersion, we use the Kaplan-Meier estimator. Because we do not see any statistical difference in the X-ray properties between the lowand high- $L_{\mathrm{bol}} / L_{\mathrm{Edd}} \mathrm{mBH}$ samples, we consider the entire 78 object $\mathrm{mBH}$ sample in the following to improve statistics. We find that the 25th-75th percentiles of the $\alpha_{\text {ox }}$ distributions for

\footnotetext{
15 About $35 \%$ of the WLQs shown in Figure 6 were initially selected as highredshift analogs to PHL 1811, which are nearly always X-ray weak and appear to share many similarities to WLQs in their optical and UV spectra. For convenience, we refer to all objects studied by Wu et al. $(2011,2012)$ and Luo et al. (2015) as WLQs.
}

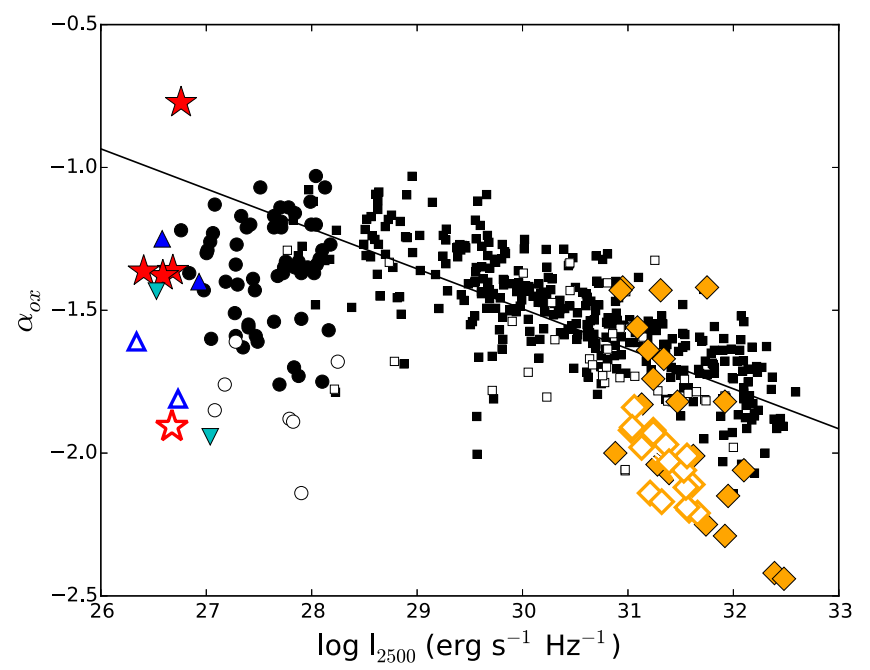

Figure 6. $\alpha_{\mathrm{ox}}$ as a function of $l_{2500}$ for our low- $L_{\mathrm{bol}} / L_{\mathrm{Edd}}$ Chandra targets (red star symbols; excluding the less-confident AGNs in galaxies 7 and 82), the four Yuan et al. (2014) low- $L_{\text {bol }} / L_{\text {Edd }}$ objects (blue triangles), the two Gültekin et al. (2014) low- $L_{\text {bol }} / L_{\text {Edd }}$ objects (cyan upside-down triangles), and the 67 objects from the high- $L_{\text {bol }} / L_{\text {Edd }} \mathrm{mBH}$ sample (circles). For comparison, we plot samples of more massive black holes, including WLQs (at $z>1.5$; orange diamonds), "normal" type-1 SDSS quasars from Just et al. (2007; squares), and the best-fit $\alpha_{\mathrm{ox}}-\log l_{2500}$ relation from Just et al. (2007; solid line). All open symbols denote upper limits on $\alpha_{\mathrm{ox}}$.

the $78 \mathrm{mBHs}$ and $38 \mathrm{WLQs}$ span a range of $0.36 \pm 0.07$ and $0.57 \pm 0.10$, respectively (errors are the standard deviations on the 25th and 75th percentile $\alpha_{\mathrm{ox}}$ values added in quadrature); when limiting the comparison Just et al. (2007) SDSS quasars to similar luminosities as the WLQ sample (120 Type-1 quasars with $l_{2500}>10^{31} \mathrm{erg} \mathrm{s}^{-1} \mathrm{~Hz}^{-1}$ ), luminous quasars have 25th75th percentiles spanning only $0.22 \pm 0.06$ in $\alpha_{\text {ox }}$. The larger dispersion hints at a potential difference in accretion properties between mBH AGNs and "normal" Type-1 quasars. In the next subsection, we consider an analogy with WLQs to explore one potential mechanism for the larger dispersion in $\alpha_{\mathrm{ox}}$, namely accretion via a slim disk.

\subsection{Comparison to Luo et al. (2015) and Slim Disk Accretion}

For $L_{\text {bol }} / L_{\text {Edd }} \gtrsim 0.1-0.3$, accretion is expected to take place in the "slim disk" regime (e.g., Abramowicz et al. 1988; Szuszkiewicz et al. 1996; Bonning et al. 2007; Straub et al. 2011), where advective cooling losses are comparable to radiative losses, and the accretion flow becomes geometrically thick and radiatively inefficient (see, e.g., Section 6 of Abramowicz \& Fragile 2013, for a brief review). Luo et al. (2015) propose that the unusual X-ray properties of WLQs (i.e., the large fraction of X-ray weak objects) may be related to accretion via slim disks, as described below.

$\mathrm{Wu}$ et al. (2011, 2012) initially suggested that WLQs contain a column of X-ray shielding gas that is very local to the black hole (interior to the broad emission line region); this $\mathrm{X}$-ray shielding gas can produce highly X-ray weak objects at certain orientations, and X-ray normal objects at other orientations. Luo et al. (2015) went on to physically associate this shielding gas with the inner edge of a (geometrically thick) slim disk. In this picture, all WLQs are fed by an inner slim disk, but only the ones oriented such that we are viewing the central engine through the "puffed up" disk material 
appear to be X-ray weak. ${ }^{16}$ For these X-ray weak objects, the direct X-ray continuum will be highly absorbed and any detected X-ray emission should be dominated by reflected/ scattered light (Luo et al. 2015). This scenario results in the population of WLQs as a whole displaying a large observed dispersion in $\alpha_{\mathrm{ox}}$.

It is tempting to appeal to a similar scenario to explain the large dispersion in $\alpha_{\mathrm{ox}}$ observed for $\mathrm{mBHs}$. However, if such a scenario were to apply to $\mathrm{mBH}$ AGN, then we expect to see a systematic change in the X-ray properties of the 78-object $\mathrm{mBH}$ sample around $L_{\mathrm{bol}} / L_{\text {Edd }} \approx 0.1-0.3$, as the accretion flow transitions between a geometrically thick slim disk and a geometrically thin (radiatively efficient) Shakura \& Sunyaev (1973) disk. In particular, we should see less dispersion in $\alpha_{\text {ox }}$ at $L_{\text {bol }} / L_{\text {Edd }} \lesssim 0.1-0.3$, in the context of a scenario where the $\mathrm{X}$-rays at these lower Eddington ratios originate from inverse Compton scattering of disk UV photons off a hot corona that is energetically coupled to a thin disk (e.g., Haardt \& Maraschi 1993). For $L_{\text {bol }} / L_{\text {Edd }} \lesssim 0.1-0.3$, the higher UV flux from the thin disk would increase the number of inverse Compton scatterings, thereby cooling the corona and also producing mildly steeper X-ray spectra on average (e.g., Ghisellini \& Haardt 1994).

A systematic change in the X-ray properties, as described above, is not observed among the $\mathrm{mBH}$ sample. However, when comparing $\log L_{\text {bol }} / L_{\text {Edd }}$ estimates from different methods in Table 6, we cannot exclude the possibility that the Eddington ratios adopted for both the low- and high- $L_{\text {bol }} / L_{\text {Edd }}$ $\mathrm{mBH}$ samples are systematically underestimated, perhaps by up to an order of magnitude. If so, then nearly all of the high$L_{\text {bol }} / L_{\text {Edd }}$ sample would be in the super-Eddington regime, and several of the lower- $L_{\text {bol }} / L_{\text {Edd }}$ objects would fall close to the proposed "slim disk" transition. Furthermore, we could also be systematically underestimating $L_{\mathrm{bol}} / L_{\mathrm{Edd}}$ for our Chandra targets if their virial-based $M_{\mathrm{BH}}$ estimates happen to be too large. Thus, we cannot exclude the possibility that the entire $\mathrm{mBH}$ AGN sample could feasibly be more rapidly accreting than they appear to be in Figure 5, in which case WLQs may provide useful insights into understanding accretion onto the $\mathrm{mBH}$ sample considered here. We note that limited information on Eddington ratios for WLQs seems to point toward $L_{\text {bol }} / L_{\text {Edd }}>0.3$; (Shemmer et al. 2010; Luo et al. 2015; Plotkin et al. 2015).

Our X-ray stacking analysis of the X-ray non-detected mBHs yields a relatively hard $\mathrm{X}$-ray $\operatorname{spectrum}(\Gamma \approx 1)$. For the objects with small $\alpha_{\mathrm{ox}}$, the inner edge of the slim disk modifies the "intrinsic" X-rays associated with the AGN corona, and any observed X-rays are likely dominated by reflection/scattering, such that a hard X-ray spectrum is expected. Among WLQs, an $\mathrm{X}$-ray stacking analysis of the subpopulation of X-ray weak objects reveals a hard X-ray spectrum as well $\left(\Gamma=1.16_{-0.32}^{+0.37}\right.$; Luo et al. 2015, also see $\mathrm{Wu}$ et al. 2012). The similarly hard (average) spectra of the subsets of X-ray weak mBH AGNs and X-ray weak WLQs may futher support a WLQ analogy. Of course, the error bars on $\Gamma$ for our stacked $\mathrm{mBHs}$ are large (see Table 5). Still, we find this to be an intriguing result, motivating a need for tighter X-ray spectral constraints for a sample of

\footnotetext{
${ }^{16}$ We stress that shielding from a geometrically thick slim disk is just one potential explanation for the WLQ phenomenon. Other ideas range from quenched X-ray coronae to evolutionary effects to gas deficient and/or multizone broad emission line regions (see, e.g., Leighly et al. 2007b; Hryniewicz et al. 2010; Laor \& Davis 2011; Liu \& Zhang 2011; Bañados et al. 2014; Plotkin et al. 2015; Shemmer \& Lieber 2015).
}

mBH AGNs spanning both high- and low- $\alpha_{\text {ox }}$, in order to rigorously compare $\Gamma$ as a function of $\alpha_{\mathrm{ox}}$.

If the WLQ analogy holds, then we require samples of $\mathrm{mBHs}$ that are accreting even more weakly than our low- $L_{\mathrm{bol}} / L_{\mathrm{Edd}}$ sample to search for a slim-to-thin disk transition by searching for systematic changes in the X-ray properties described earlier (i.e., less dispersion in $\alpha_{\mathrm{ox}}$ and steeper $\Gamma$ at lower Eddington ratios in the thin disk regime). The prototype $\mathrm{mBH}$ NGC 4395 (Filippenko 1989) has a well-determined bolometric luminosity $L_{\text {bol }}=5.3 \times 10^{40} \mathrm{erg} \mathrm{s}^{-1}$ from a highly sampled broadband spectrum (Moran et al. 2005), providing $L_{\mathrm{bol}} / L_{\mathrm{Edd}} \sim 0.001$ for $M_{\mathrm{BH}}=(3.6 \pm 1.1) \times 10^{5} M_{\odot}$ (Peterson et al. 2005). Intriguingly, NGC 4395 is not only accreting below the expected slimto-thin disk transition at $0.1-0.3 L_{\text {bol }} / L_{\text {Edd }}$, but it is also near or below another critical accretion regime at $\sim 0.01 L_{\text {Edd }}$, where the disk is expected to switch from a thin disk to a radiatively inefficient accretion flow (RIAF), ${ }^{17}$ as described below.

For AGNs fed by a thin disk, the X-ray photon index $\Gamma$ is correlated with Eddington ratio when $L_{\text {bol }} / L_{\text {Edd }} \gtrsim 0.01$ (e.g., Shemmer et al. 2008; Risaliti et al. 2009; Brightman et al. 2013). Below $\sim 0.01 L_{\text {Edd }}, \Gamma$ and $L_{\text {bol }} / L_{\text {Edd }}$ are anti-correlated, so that AGNs show the hardest X-ray spectra around $L_{\text {bol }} / L_{\text {Edd }} \sim 0.01$, which may indicate a transition from a thin disk to a RIAF around 1\% L Edd (e.g., Constantin et al. 2009; Gu \& Cao 2009; Younes et al. 2011; Trichas et al. 2013; note that $\Gamma$ eventually plateaus to $\Gamma \sim 2.1$ at the lowest Eddington ratios, e.g., Yang et al. 2015). This X-ray spectral behavior is observed for stellar mass black holes in XRB systems as well (e.g., Esin et al. 1997; Tomsick et al. 2001; Wu \& Gu 2008; Sobolewska et al. 2011; Plotkin et al. 2013; Yang et al. 2015), suggesting that it is a universal feature of black hole accretion, regardless of black hole mass. NGC 4395 supports this trend for mBHs, because it displays a hard photon index $\Gamma=0.61 \pm 0.15$ at $L_{\text {bol }} / L_{\text {Edd }} \approx 10^{-3}$ (Moran et al. 2005). We stress that we have no reason to suspect that the bolometric luminosity of NGC 4395 could be biased in the same manner as for the other mBHs (because its $L_{\text {bol }}$ is calculated from an observed broadband spectrum of the nucleus). The physical mechanism for the small $\Gamma$ for NGC 4395 is therefore different than the small $\Gamma$ discussed earlier in the context of a slim disk. This intriguing trend is far from robust, as it is based on a single source, and it further motivates a need for high signal-to-noise X-ray spectra for $\mathrm{mBHs}$ across a wide range of $L_{\mathrm{bol}} / L_{\mathrm{Edd}}$.

A sizeable population of lower- $L_{\mathrm{bol}} / L_{\mathrm{Edd}} \mathrm{mBH}$ AGNs is unlikely accessible from optical-selection techniques, however, and recovering such objects will require complementary multiwavelength searches. High spatial resolution X-ray surveys (especially when combined with the radio) are a promising avenue for revealing weakly accreting black holes (e.g., Soria et al. 2006; Gallo et al. 2010; Pellegrini 2010; Reines et al. 2011, 2014; Reines \& Deller 2012; Lemons et al. 2015; Miller et al. 2015; also note that galaxies 53 and 56 in the current work show X-ray signatures of an AGN, but lack optical photoionization signatures of activity). Success has also

\footnotetext{
${ }^{17}$ We stress that while high-accretion rate slim disks $\left(L_{\text {bol }} / L_{\text {Edd }} \gtrsim 0.1-0.3\right)$ and low-accretion rate RIAFs $\left(L_{\text {bol }} / L_{\text {Edd }} \lesssim 0.01\right)$ are both radiatively inefficient, the physical reasons for their radiative inefficiencies are quite different. Slim disks are radiatively inefficient largely because of photon trapping effects at near-Eddington luminosities; in most low-accretion rate RIAF models, the low radiative efficiency is mainly due to weak Coulomb coupling in the accretion flow (e.g., Ichimaru 1977; Narayan \& Yi 1994; Abramowicz et al. 1995). For other variants of RIAFs at low-accretion rates, see, e.g., Blandford \& Begelman (1999), Narayan et al. (2000), Quataert \& Gruzinov (2000), and Merloni \& Fabian (2002).
} 
been achieved through infrared surveys (e.g., Satyapal et al. 2008) and fast variability (Kamizasa et al. 2012; Ho \& Kim 2016; Morokuma et al. 2016).

We stress that it is unclear whether the optically based $L_{\text {bol }} / L_{\text {Edd }}$ measurements are systematically underestimated, and the WLQ analogy is only meant to represent one possibility. If the adopted $L_{\mathrm{bol}} / L_{\mathrm{Edd}}$ estimates are accurate, then there does not appear to be a distinct Eddington ratio that marks a transition in radiative efficiency. In that case, a range of accretion disk/corona properties may contribute to the dispersion in $\alpha_{\text {ox }}$, which may include a substantial number of intrinsically X-ray weak AGN. To confirm or refute current $L_{\text {bol }} / L_{\text {Edd }}$ estimates, broadband spectral energy distributions for a substantially larger number of $\mathrm{mBHs}$ are required to properly constrain the bolometric corrections for this population. Such a project will require high-resolution imaging across the entire electromagnetic spectrum to separate the AGNs from the host galaxies (see D12 for further discussion, as well as, e.g., Moran et al. 1999, 2005; Thornton et al. 2008; Constantin \& Seth 2012, for examples of well-sampled broadband spectra).

\section{CONCLUSIONS}

We find no evidence for a difference in the X-ray properties of mBHs from $-2<\log L_{\text {bol }} / L_{\text {Edd }}<0$. We argue that either (1) optically based $L_{\text {bol }} / L_{\text {Edd }}$ estimates are systematically underestimated and nearly all $\mathrm{mBHs}$ accrete from a geometrically thick, radiatively inefficient slim disk; or (2) there is variety in the accretion details among individual objects, but there is no evidence for a systematic change in accretion properties at a specific Eddington ratio. If $\mathrm{mBHs}$ do accrete from a slim disk, then super-Eddington accretion could provide a mechanism for growing SMBHs in the early universe (e.g., Madau et al. 2014). Finally, Madau \& Haardt (2015) recently showed that a faint high-redshift AGN population could produce enough flux to account for the epoch of reionization, provided that AGN X-ray-to-UV luminosity ratios in the early universe are not too hard (Madau \& Haardt 2015 explicitly adopt $\alpha_{\mathrm{ox}} \approx-1.4$ ). From an empirical perspective (and regardless of the accretion mode), the observed flattening of the $\alpha_{\mathrm{ox}}-l_{2500}$ relation at low luminosities implies that accretion onto $\mathrm{mBHs}$ produces a broadband continuum in line with requirements for $\mathrm{AGN}$-driven reioniziation.

We thank the referee for suggestions that improved the presentation of this paper. We thank Ohad Shemmer for helpful discussions, and Anna Conlon for advice on our survival analysis. Support for this work was provided by the National Aeronautics and Space Administration through Chandra Award Number GO3-14116X issued by the Chandra X-ray Observatory Center, which is operated by the Smithsonian Astrophysical Observatory for and on behalf of the National Aeronautics Space Administration under contract NAS803060. Support for AER was provided by NASA through Hubble Fellowship grant HST-HF2-51347.001-A awarded by the Space Telescope Science Institute, which is operated by the Association of Universities for Research in Astronomy, Inc., for NASA, under contract NAS 5-26555. This research has made use of software provided by the Chandra X-ray Center $(\mathrm{CXC})$ in the application package CIAO. This research has made use of data obtained from the Chandra Data Archive, and software provided by the Chandra X-Ray Center (CXC) in the application packages CIAO, ChIPS, and Sherpa.

\section{REFERENCES}

Abramowicz, M. A., Chen, X., Kato, S., Lasota, J.-P., \& Regev, O. 1995, ApJL, 438, L37

Abramowicz, M. A., Czerny, B., Lasota, J. P., \& Szuszkiewicz, E. 1988, ApJ, 332,646

Abramowicz, M. A., \& Fragile, P. C. 2013, LRR, 16, 1

Avni, Y., \& Tananbaum, H. 1982, ApJL, 262, L17

Baldassare, V. F., et al. 2016, ApJ, in press

Baldassare, V. F., Reines, A. E., Gallo, E., \& Greene, J. E. 2015, ApJL, 809, L14

Baldwin, J. A., Phillips, M. M., \& Terlevich, R. 1981, PASP, 93, 5

Bañados, E., Venemans, B. P., Morganson, E., et al. 2014, AJ, 148, 14

Barth, A. J., Greene, J. E., \& Ho, L. C. 2008, AJ, 136, 1179

Barth, A. J., Ho, L. C., Rutledge, R. E., \& Sargent, W. L. W. 2004, ApJ, 607, 90

Begelman, M. C. 2002, ApJL, 568, L97

Begelman, M. C., Volonteri, M., \& Rees, M. J. 2006, MNRAS, 370, 289

Blandford, R. D., \& Begelman, M. C. 1999, MNRAS, 303, L1

Bonning, E. W., Cheng, L., Shields, G. A., Salviander, S., \& Gebhardt, K. 2007, ApJ, 659, 211

Brightman, M., Silverman, J. D., Mainieri, V., et al. 2013, MNRAS, 433, 2485

Bromm, V., Coppi, P. S., \& Larson, R. B. 2002, ApJ, 564, 23

Cardelli, J. A., Clayton, G. C., \& Mathis, J. S. 1989, ApJ, 345, 245

Cash, W. 1979, ApJ, 228, 939

Constantin, A., Green, P., Aldcroft, T., et al. 2009, ApJ, 705, 1336

Constantin, A., \& Seth, A. C. 2012, AdAst, 2012, 178060

Cowie, L. L., Barger, A. J., \& Hasinger, G. 2012, ApJ, 748, 50

Davis, J. E. 2001, ApJ, 562, 575

Desroches, L.-B., Greene, J. E., \& Ho, L. C. 2009, ApJ, 698, 1515

Dewangan, G. C., Mathur, S., Griffiths, R. E., \& Rao, A. R. 2008, ApJ, 689, 762

Diamond-Stanic, A. M., Fan, X., Brandt, W. N., et al. 2009, ApJ, 699, 782

Dickey, J. M., \& Lockman, F. J. 1990, ARA\&A, 28, 215

Done, C., Davis, S. W., Jin, C., Blaes, O., \& Ward, M. 2012, MNRAS, 420, 1848

Dong, R., Greene, J. E., \& Ho, L. C. 2012a, ApJ, 761, 73 (D12)

Dong, X.-B., Ho, L. C., Yuan, W., et al. 2012b, ApJ, 755, 167

Esin, A. A., McClintock, J. E., \& Narayan, R. 1997, ApJ, 489, 865

Fan, X., Strauss, M. A., Gunn, J. E., et al. 1999, ApJL, 526, L57

Fan, X., Strauss, M. A., Richards, G. T., et al. 2006, AJ, 131, 1203

Feigelson, E. D., \& Nelson, P. I. 1985, ApJ, 293, 192

Feng, H., \& Soria, R. 2011, NewAR, 55, 166

Filippenko, A. V. 1989, AJ, 97, 726

Filippenko, A. V., \& Ho, L. C. 2003, ApJL, 588, L13

Fruscione, A., McDowell, J. C., Allen, G. E., et al. 2006, Proc. SPIE, 6270, $62701 \mathrm{v}$

Gallo, E., Treu, T., Marshall, P. J., et al. 2010, ApJ, 714, 25

Garmire, G. P., Bautz, M. W., Ford, P. G., Nousek, J. A., \& Ricker, G. R., Jr. 2003, Proc. SPIE, 4851, 28

Gehrels, N. 1986, ApJ, 303, 336

Ghisellini, G., \& Haardt, F. 1994, ApJL, 429, L53

Gilfanov, M. 2004, MNRAS, 349, 146

Greene, J. E. 2012, NatCo, 3, 1304

Greene, J. E., \& Ho, L. C. 2004, ApJ, 610, 722

Greene, J. E., \& Ho, L. C. 2005, ApJ, 630, 122

Greene, J. E., \& Ho, L. C. 2007a, ApJ, 670, 92 (GH07)

Greene, J. E., \& Ho, L. C. 2007b, ApJ, 667, 131

Greene, J. E., \& Ho, L. C. 2007c, ApJ, 656, 84

Grimm, H.-J., Gilfanov, M., \& Sunyaev, R. 2003, MNRAS, 339, 793

Gu, M., \& Cao, X. 2009, MNRAS, 399, 349

Gültekin, K., Cackett, E. M., King, A. L., Miller, J. M., \& Pinkney, J. 2014, ApJL, 788, L22

Haardt, F., \& Maraschi, L. 1993, ApJ, 413, 507

Halpern, J. P., \& Steiner, J. E. 1983, ApJL, 269, L37

Ho, L., \& Kim, M. 2016, ApJ, 821, 48

Houck, J. C., \& Denicola, L. A. 2000, in ASP Conf. Ser. 216, Astronomical Data Analysis Software and Systems IX, ed. N. Manset, C. Veillet, \& D. Crabtree (San Francisco, CA: ASP), 591

Hryniewicz, K., Czerny, B., Nikołajuk, M., \& Kuraszkiewicz, J. 2010, MNRAS, 404, 2028

Ichimaru, S. 1977, ApJ, 214, 840

Irwin, J. A., Bregman, J. N., \& Athey, A. E. 2004, ApJL, 601, L143

Jiang, Y.-F., Greene, J. E., Ho, L. C., Xiao, T., \& Barth, A. J. 2011, ApJ, 742,68

Just, D. W., Brandt, W. N., Shemmer, O., et al. 2007, ApJ, 665, 1004 
Kamizasa, N., Terashima, Y., \& Awaki, H. 2012, ApJ, 751, 39

Kauffmann, G., \& Heckman, T. M. 2009, MNRAS, 397, 135

Kauffmann, G., Heckman, T. M., Tremonti, C., et al. 2003, MNRAS, 346, 1055

Kelly, B. C. 2007, ApJ, 665, 1489

Kelly, B. C., Bechtold, J., Trump, J. R., Vestergaard, M., \& Siemiginowska, A. 2008, ApJS, 176, 355

Kewley, L. J., Dopita, M. A., Sutherland, R. S., Heisler, C. A., \& Trevena, J. 2001, ApJ, 556, 121

Kewley, L. J., Groves, B., Kauffmann, G., \& Heckman, T. 2006, MNRAS, 372,961

King, A. R. 2002, MNRAS, 335, L13

Kormendy, J., \& Ho, L. C. 2013, ARA\&A, 51, 511

Kraft, R. P., Burrows, D. N., \& Nousek, J. A. 1991, ApJ, 374, 344

Lane, R. A., Shemmer, O., Diamond-Stanic, A. M., et al. 2011, ApJ, 743, 163

Laor, A., \& Davis, S. W. 2011, MNRAS, 417, 681

Lavalley, M., Isobe, T., \& Feigelson, E. 1992, in ASP Conf. Ser. 25, Astronomical Data Analysis Software and Systems I, ed. D. M. Worrall, C. Biemesderfer, \& J. Barnes (San Francisco, CA: ASP), 245

Leighly, K. M., Halpern, J. P., Jenkins, E. B., \& Casebeer, D. 2007a, ApJS, 173,1

Leighly, K. M., Halpern, J. P., Jenkins, E. B., et al. 2007b, ApJ, 663, 103

Lemons, S. M., Reines, A. E., Plotkin, R. M., Gallo, E., \& Greene, J. E. 2015, ApJ, 805, 12

Liu, Y., \& Zhang, S. N. 2011, ApJL, 728, L44

Lodato, G., \& Natarajan, P. 2006, MNRAS, 371, 1813

Ludlam, R. M., Cackett, E. M., Gültekin, K., et al. 2015, MNRAS, 447, 2112

Luo, B., Brandt, W. N., Hall, P. B., et al. 2015, ApJ, 805, 122

Madau, P., \& Haardt, F. 2015, ApJL, 813, L8

Madau, P., Haardt, F., \& Dotti, M. 2014, ApJL, 784, L38

Madau, P., \& Rees, M. J. 2001, ApJL, 551, L27

Maoz, D. 2007, MNRAS, 377, 1696

McLure, R. J., \& Dunlop, J. S. 2004, MNRAS, 352, 1390

Merloni, A., \& Fabian, A. C. 2002, MNRAS, 332, 165

Miller, B. P., Gallo, E., Greene, J. E., et al. 2015, ApJ, 799, 98

Mineo, S., Gilfanov, M., Lehmer, B. D., Morrison, G. E., \& Sunyaev, R. 2014, MNRAS, 437, 1698

Miniutti, G., Ponti, G., Greene, J. E., et al. 2009, MNRAS, 394, 443

Moran, E. C., Eracleous, M., Leighly, K. M., et al. 2005, AJ, 129, 2108

Moran, E. C., Filippenko, A. V., Ho, L. C., et al. 1999, PASP, 111, 801

Moran, E. C., Shahinyan, K., Sugarman, H. R., Vélez, D. O., \& Eracleous, M. 2014, AJ, 148, 136

Morokuma, T., Tominaga, N., Tanaka, M., et al. 2016, PASJ, 68, 40

Narayan, R., Igumenshchev, I. V., \& Abramowicz, M. A. 2000, ApJ, 539, 798

Narayan, R., \& Yi, I. 1994, ApJL, 428, L13

Natarajan, P. 2014, GReGr, 46, 1702

Pellegrini, S. 2010, ApJ, 717, 640

Peterson, B. M., Bentz, M. C., Desroches, L.-B., et al. 2005, ApJ, 632, 799

Plotkin, R. M., Anderson, S. F., Brandt, W. N., et al. 2010a, ApJ, 721, 562

Plotkin, R. M., Anderson, S. F., Brandt, W. N., et al. 2010b, AJ, 139, 390
Plotkin, R. M., Gallo, E., \& Jonker, P. G. 2013, ApJ, 773, 59

Plotkin, R. M., Gallo, E., Miller, B. P., et al. 2014, ApJ, 780, 6

Plotkin, R. M., Shemmer, O., Trakhtenbrot, B., et al. 2015, ApJ, 805, 123

Poutanen, J., Lipunova, G., Fabrika, S., Butkevich, A. G., \& Abolmasov, P. 2007, MNRAS, 377, 1187

Quataert, E., \& Gruzinov, A. 2000, ApJ, 539, 809

Reines, A. E., \& Deller, A. T. 2012, ApJL, 750, L24

Reines, A. E., Greene, J. E., \& Geha, M. 2013, ApJ, 775, 116

Reines, A. E., Plotkin, R. M., Russell, T. D., et al. 2014, ApJL, 787, L30

Reines, A. E., Sivakoff, G. R., Johnson, K. E., \& Brogan, C. L. 2011, Natur, 470, 66

Reines, A. E., \& Volonteri, M. 2015, ApJ, 813, 82

Ricci, C., Paltani, S., Ueda, Y., \& Awaki, H. 2013, MNRAS, 435, 1840

Risaliti, G., Young, M., \& Elvis, M. 2009, ApJL, 700, L6

Satyapal, S., Vega, D., Dudik, R. P., Abel, N. P., \& Heckman, T. 2008, ApJ, 677, 926

Schramm, M., Silverman, J. D., Greene, J. E., et al. 2013, ApJ, 773, 150

Shakura, N. I., \& Sunyaev, R. A. 1973, A\&A, 24, 337

Shemmer, O., Brandt, W. N., Anderson, S. F., et al. 2009, ApJ, 696, 580

Shemmer, O., Brandt, W. N., Netzer, H., Maiolino, R., \& Kaspi, S. 2008, ApJ, 682,81

Shemmer, O., \& Lieber, S. 2015, ApJ, 805, 124

Shemmer, O., Trakhtenbrot, B., Anderson, S. F., et al. 2010, ApJL, 722, L152

Sobolewska, M. A., Papadakis, I. E., Done, C., \& Malzac, J. 2011, MNRAS, 417,280

Soria, R., Fabbiano, G., Graham, A. W., et al. 2006, ApJ, 640, 126

Spergel, D. N., Verde, L., Peiris, H. V., et al. 2003, ApJS, 148, 175

Steffen, A. T., Strateva, I., Brandt, W. N., et al. 2006, AJ, 131, 2826

Straub, O., Bursa, M., Sądowski, A., et al. 2011, A\&A, 533, A67

Swartz, D. A., Soria, R., Tennant, A. F., \& Yukita, M. 2011, ApJ, 741, 49

Szuszkiewicz, E., Malkan, M. A., \& Abramowicz, M. A. 1996, ApJ, 458, 474

Tananbaum, H., Avni, Y., Branduardi, G., et al. 1979, ApJL, 234, L9

Thornton, C. E., Barth, A. J., Ho, L. C., Rutledge, R. E., \& Greene, J. E. 2008, ApJ, 686, 892

Tomsick, J. A., Corbel, S., \& Kaaret, P. 2001, ApJ, 563, 229

Trichas, M., Green, P. J., Constantin, A., et al. 2013, ApJ, 778, 188

Vanden Berk, D. E., Richards, G. T., Bauer, A., et al. 2001, AJ, 122, 549

Walton, D. J., Roberts, T. P., Mateos, S., \& Heard, V. 2011, MNRAS, 416, 1844

Willott, C. J. 2011, ApJL, 742, L8

Willott, C. J., McLure, R. J., \& Jarvis, M. J. 2003, ApJL, 587, L15

Wu, J., Brandt, W. N., Anderson, S. F., et al. 2012, ApJ, 747, 10

Wu, J., Brandt, W. N., Hall, P. B., et al. 2011, ApJ, 736, 28

Wu, Q., \& Gu, M. 2008, ApJ, 682, 212

Wu, X.-B., Wang, F., Fan, X., et al. 2015, Natur, 518, 512

Yang, Q.-X., Xie, F.-G., Yuan, F., et al. 2015, MNRAS, 447, 1692

York, D. G., Adelman, J., Anderson, J. E., Jr., et al. 2000, AJ, 120, 1579

Younes, G., Porquet, D., Sabra, B., \& Reeves, J. N. 2011, A\&A, 530, A149

Yuan, W., Zhou, H., Dou, L., et al. 2014, ApJ, 782, 55 